\title{
Faire pâturer, Faire société, durablement
}

Éleveurs et élevages, entre inscription territoriale et changements globaux

Grazing, building society... sustainably. Farmers and farming, between

territorial identity and global changes

Lucie Dupré, Jacques Lasseur et René Poccard-Chapuis

\section{(2) OpenEdition}

Journals

Édition électronique

URL : https://journals.openedition.org/tc/7492

DOI : $10.4000 /$ tc. 7492

ISBN : 0248-6016

ISSN : 1952-420X

Éditeur

Éditions de l'EHESS

\section{Édition imprimée}

Date de publication : 1 mai 2015

Pagination : 202-231

ISBN : 9782713224874

ISSN : 0248-6016

Référence électronique

Lucie Dupré, Jacques Lasseur et René Poccard-Chapuis, « Faire pâturer, Faire société, durablement », Techniques \& Culture [En ligne], 63 | 2015, mis en ligne le 21 décembre 2018, consulté le 29 septembre 2022. URL : http://journals.openedition.org/tc/7492 ; DOI : https://doi.org/10.4000/tc.7492 


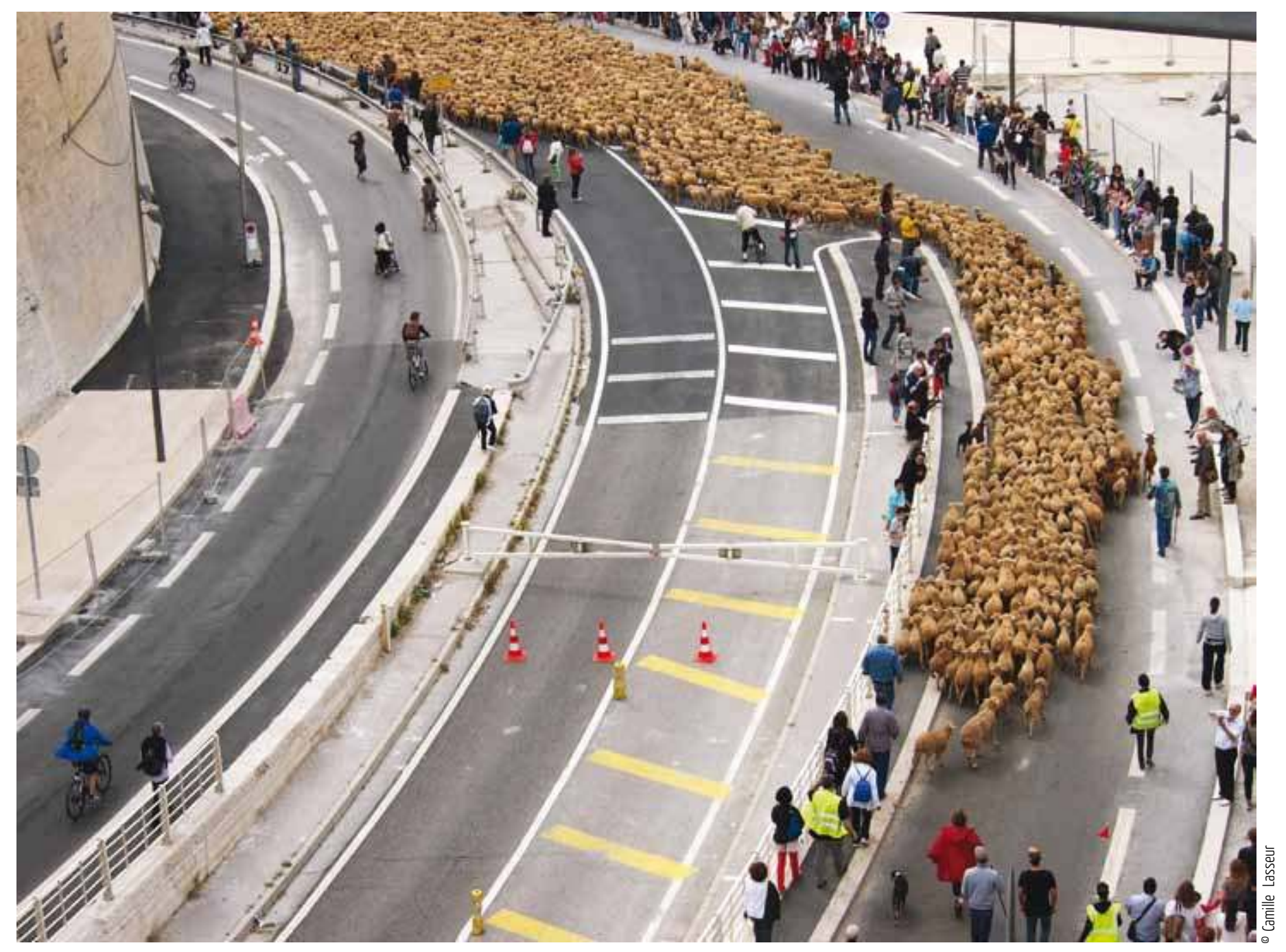




\section{\& René Poccard-Chapuis}

INRA; INRA; CIRAD

lucie.dupre@ivry.inra.fr; lasseur@supagro.inra.fr ;

rene.poccard-chapuis@cirad.frinrap.fr

\section{FAIRE PÂTURER, FAIRE SOCIÉTÉ, DURABLEMENT}

\section{Éleveurs et élevages, entre inscription territoriale et changements globaux}

En 1968, G. Hardin publie « la tragédie des communs », texte qui sera longuement, âprement, fréquemment discuté, commenté, critiqué. C'est sur l'usage collectif des pâturages qu'il prend appui en attirant l'attention sur ses conséquences en termes d'épuisement des ressources naturelles, dans un monde de plus en plus peuplé (Hardin, 1968). F. Lochet voit dans ce texte la "tragédie de la finitude», finitude des ressources et des territoires que les clichés de la terre pris par les missions Appolo, dans les années 1970, sont venus illustrer. Cette prise de conscience collective se fait dans un contexte de Guerre Froide mettant au cœur du débat public le pouvoir des États, son affaiblissement ou son affirmation (Lochet, 2014). Presque 50 ans plus tard, les mêmes inquiétudes auxquelles s'en ajoutent de nouvelles - sont formulées à des niveaux assurément plus critiques: croissance démographique, érosion des sols, épuisement des ressources fossiles et hydriques, crises énergétique et alimentaire, changement climatique, extinction d'espèces, gaspillage... Elles s'amplifient, deviennent impérieuses, occupent le cœur de débats publics - l'organisation de la conférence internationale sur le changement climatique, à Paris fin 2015, en témoigne notamment. Les problèmes sont reformulés et les questions déplacées à l'échelle de la planète: outre les intérêts nationaux, il faut nourrir le monde, lutter contre le changement climatique global, gérer durablement nos écosystèmes. C'est dans ce jardin planétaire que, face à de tels défis, les activités agricoles sont en train de se transformer profondément (Caron $\&$ al., 2013).

Le rapport Brundtland a défini, en 1987, le développement durable comme la capacité à s'engager dans des activités qui reposent sur les piliers environnemental, économique et social. Une sorte de nouveau contrat fictif était ainsi établi, notamment pour les activités agricoles qui sont particulièrement concernées puisqu'elles reposent sur un rapport de 
production, donc social et économique, s'appuyant sur des ressources naturelles. Le développement durable sera officiellement intronisé comme objectif collectif global lors du Sommet de la terre à Rio en 1992. Les conférenciers le théorisent comme un développement répondant « aux besoins du présent sans compromettre la capacité des générations futures », un développement économiquement efficace, socialement équitable et écologiquement soutenable. Les années 2000 ouvrent une période de bilans dressés dans le cadre d'expertises collectives conduites à l'échelle mondiale. L'agriculture, à la confluence de l'économie et de l'écologie, y occupe une place prépondérante. Trois grands rapports feront date. En 2005, l'évaluation des écosystèmes pour le millénaire (le Millenium Ecosystem Assesment, dit MEA) commandé par l'Organisation des Nations Unies (ONU), a mobilisé plus de 1500 experts (MEA, 2005). Il envisage les écosystèmes en lien avec « le bien être humain » (human well being) sous un angle très particulier, celui des «services écosystémiques ». Le MEA introduit une façon nouvelle de parler des relations nature-sociétés, empruntant largement au répertoire des sciences économiques. À travers la notion de « services écosystémiques», l'environnement est évalué à l'aune des services qu'il rend aux hommes. Cette approche a largement essaimé dans les communautés scientifiques, où la notion de «service » s'est substituée à celle de « biodiversité » faisant évoluer de façon significative la façon de concevoir le rapport à la nature (Maris, 2014). Le paradigme économique se conforte lentement et nourrit un point de vue global sur les défis de l'agriculture: l'agriculture doit nourrir la planète en étant « performante » écologiquement et économiquement. La notion d'intensification, pourtant responsable des dégâts environnementaux et sociaux, refait surface en Europe au motif d'une meilleure efficience dans l'usage de ressources pas toujours renouvelables dans des territoires, quant à eux, finis. Quant au second rapport, nous l'avons évoqué dans l'introduction de ce Thema. Exclusivement dédié à l'élevage, il s'agit du Livestock long shadow que l'Organisation des Nations Unies pour l'Alimentation et l'Agriculture publie en 2006, sous l'égide de la FAO (Steinfeld E al., 2006). Nous avons déjà souligné la forte critique que ce rapport déployait à l'encontre de certains types d'élevage, notamment en termes de destruction des écosystèmes, des ressources stratégiques et des sols; il sera suivi, en 2009, d'un autre rapport nettement moins à charge, Livestock in a changing landscape (Steinfeld E al., 2009). Enfin, finalisée en 2008 après avoir été lancée par la FAO et la Banque mondiale, une expertise collective internationale menée durant quatre ans appelée « l'IAASTD » (Évaluation internationale des sciences et technologies agricoles pour le développement). Initialement conçus comme la transposition des scénarios du Millénium, ses objectifs ont évolué vers l'analyse de l'impact des méthodes de production sur l'environnement (Even, 2009). La conclusion est sans appel: la production alimentaire actuelle n'est pas durable. Si ce constat rejoint en partie les précédents, il s'en distingue aussi nettement. En effet, l'accent est porté sur des dimensions absentes ou très peu évoquées dans les deux rapports précédents. En effet, d'une part, il est pointé que l'agriculture, multifonctionnelle et multifactorielle, demande des approches prenant en compte les spécificités des situations locales, notamment des savoirs; d'autre part, la vulnérabilité sociale est placée non plus en périphérie mais au cœur de la réflexion et des recommandations.

Suite à ces bilans, le pâturage est fortement questionné dans un sens qui a significativement évolué. Les enjeux liés au pâturage et aux activités d'élevage sont au cœur de ces changements d'échelles spatiales et temporelles, par lesquelles s'exprime une mondialisation à la fois économique et écologique. Ils se trouvent, plus que jamais, pris en tension 
entre écologie et économie, deux domaines a priori distincts mais dont les frontières tendent à se brouiller entre d'un côté, le verdissement des pratiques agricoles par le biais des politiques publiques (Deverre \& de Sainte Marie, 2008), et de l'autre, la marchandisation de la nature (Maris, 2014). L'élevage et le pâturage sont ainsi nouvellement questionnés, à l'aune du préjudice qu'ils causent sur l'environnement (et comment le limiter) comme à celle de leur contribution au bon fonctionnement des écosystèmes. L'élevage à l'herbe est-il en train de se tertiariser? Que reste-t-il de sa fonction nourricière? Qu'en est-il de ses hommes? En effet, si l'on retrouve bien les piliers économique et écologique, le premier se verdissant tandis que le second se financiarisant, les questions qu'ils relaient, amplifiées et reformulées, laissent peu de place au volet proprement social du développement durable ${ }^{1}$. Or, peut-il y avoir de l'élevage sans éleveurs et sans inscription dans un territoire? Le pâturage nous dit clairement que non. Des hommes, éleveurs parfois sans terre, doivent eux aussi pouvoir vivre de leur métier, dans leurs territoires, et s'y inscrire durablement. Le développement durable arguait de la nécessité de faire tenir ensemble environnement, économie et société, trois dimensions qui structurent entièrement le pâturage en des points de tensions encore complexifiées par les jeux d'échelles (du local au global, du brin d'herbe à la planète). La diversité des pâturages dans le monde, en même temps que les profonds changements qu'ils subissent, nous rappellent à quel point les enjeux économiques et écologiques ne peuvent être dans le même temps que sociaux, à quel point il est difficile, sinon périlleux, de les disjoindre sans remettre en cause la cohérence de l'ensemble. C'est donc sur les modalités, les formes et les enjeux de son ancrage - social, écologique et économique - dans ses territoires que nous reviendrons dans cet article.

Prenant appui sur les contributions du Thema, nous discutons, dans un premier temps du rapport au vivant et des savoirs que le pâturage suppose, entre activité professionnelle et services éco-systémiques. Ici, la question scalaire, des savoirs et des espaces, est centrale. Ensuite, nous nous interrogeons sur la façon dont ces activités d'élevage au pâturage évoluent plus globalement relativement à d'autres formes d'occupation du sol dans des rapports sociaux souvent de plus en plus tendus. Enfin, nous examinons comment le pâturage est une fabrique sociale de territoires, où il puise ses ressources pour s'affirmer non seulement socialement et économiquement, mais aussi culturellement.

\section{Feux de brousse en zone tropicale} humide

Togo Hills, Hohué, Ghana, 2007.

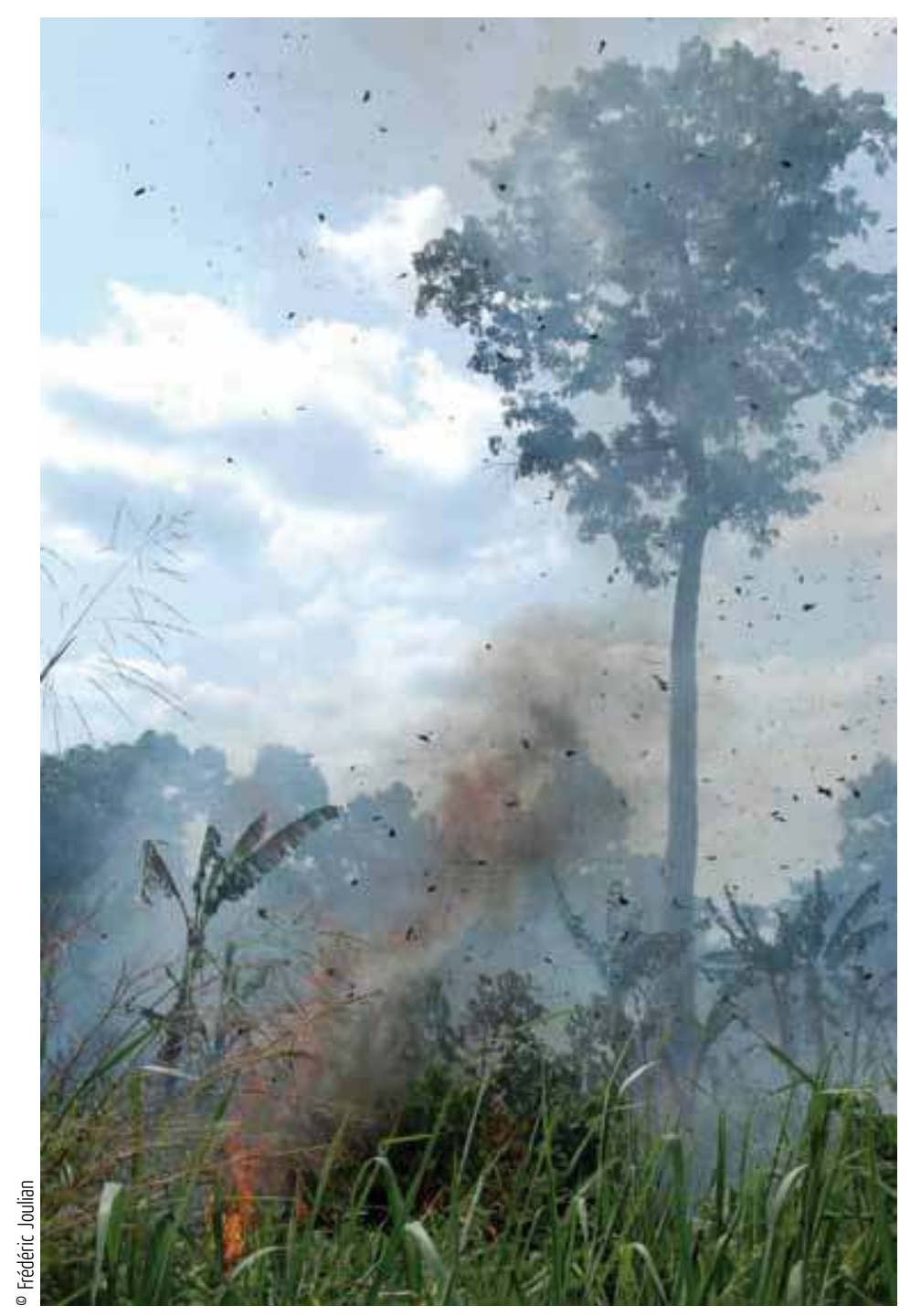




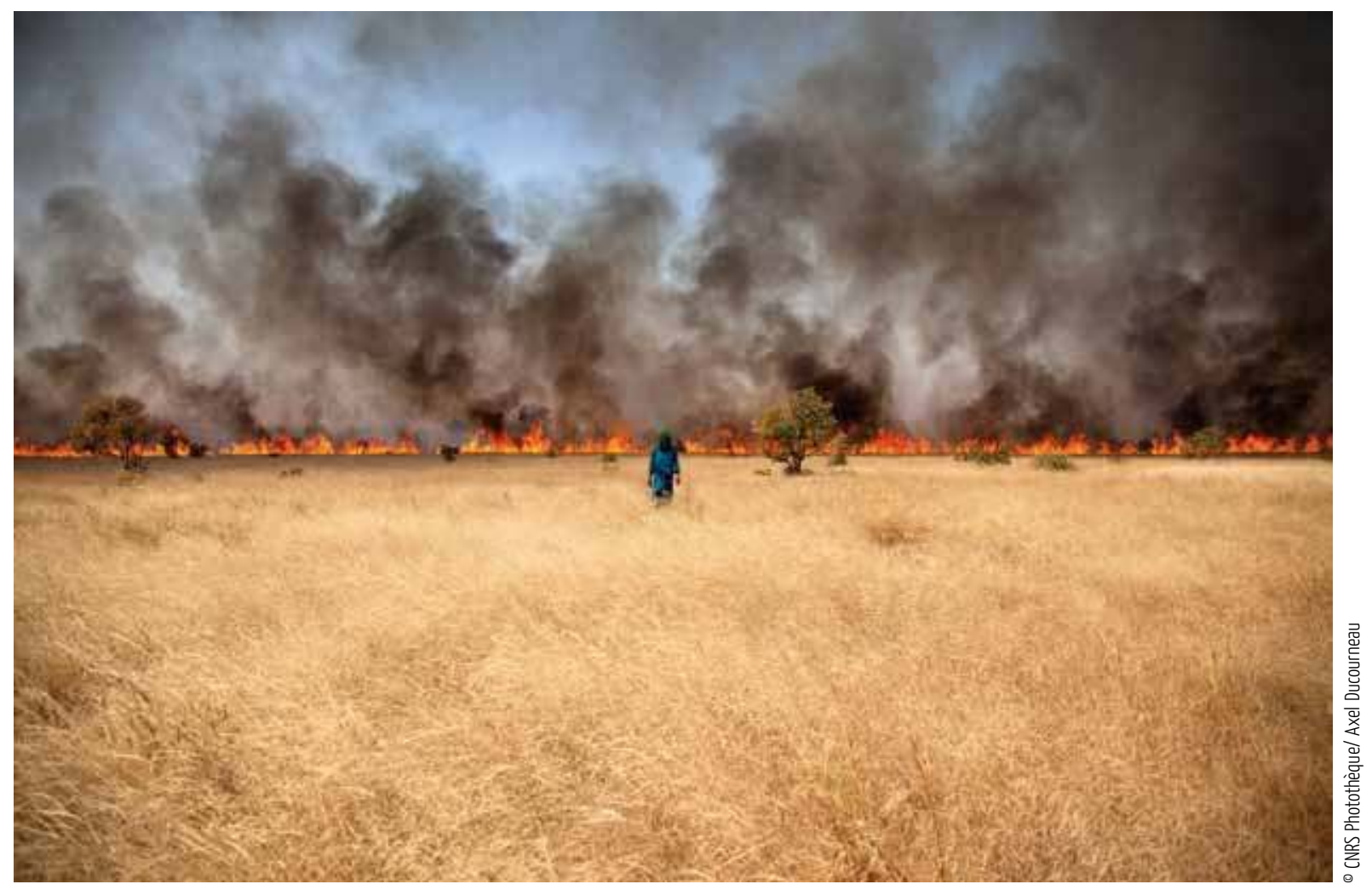

\section{Feux de brousse}

Ils sont un des fléaux de la zone sahélienne. Alimentés par les vents chauds du désert, comme l'Harmattan, et la végétation desséchée, ils peuvent détruire d'immenses surfaces de pâturages. Celui-ci, dans le Ferlo, au nord du Sénégal, s'est propagé pendant près de 10 heures, détruisant plusieurs milliers d'hectares. Le contrôle collectif du feu est un des enjeux majeurs du dialogue entre populations d'éleveurs et d'agriculteurs dans la région. Le feu n'est pas toujours un ennemi, il a des qualités aussi, mais tout est question de gestion: les feux tardifs de saison sèche sont dévastateurs, mais les feux précoces renouvellent le fourrage, nettoient les champs, etc.

\section{De la culture locale du pâturage...}

Le pâturage évoque tout de suite et avant tout la haute figure du pasteur - avec ou sans terre, parfois également éleveur - poussant son troupeau dans d'immenses territoires sauvages, prairies ou steppes d'altitude et hauts plateaux. Ces images ne sont pas que d'Épinal: le berger demeure dans les systèmes pastoraux le spécialiste de la garde des troupeaux et du milieu naturel, spécialiste emblématique et singulier qui a attiré l'attention de nombreux chercheurs (Landais \& Deffontaines, 1988; Darré \& al., 1994; Faye 2008; Meuret, 2010; Brisebarre, 1978, 2013; Brisebarre \& al., 2009; Ravis-Giordani [1983] 2001). Si la figure du pasteur s'impose d'évidence, ce volume rappelle que le pâturage, dans sa pratique comme dans ses territoires, ne concerne pas seulement le berger - lequel n'existe d'ailleurs pas, plus toujours. Le pâturage concerne également des espaces cultivés et semés, prairies temporaires ou permanentes, pour lesquels la clôture a parfois remplacé le berger (Lasseur, 2005). Dans tous les cas, entre tactique et stratégie, le travail de l'herbe est, nous l'avons vu, une œuvre complexe de gestion, d'anticipation, d'ajustement, par la constitution de stock et de réserves fourragères sur pied ou récoltés (Daget \& Godron, 1995). Différentes échelles d'espaces sont appréhendées, à autant d'échelles de temps, de la pâture itinérante quotidienne à la gestion saisonnière des différents versants ou parcours, à celle pluri annuelle de la prairie «temporaire » (Mathieu, 2004). La gestion du stock fourrager est au cœur des enjeux: le constituer au bon moment pour optimiser la valeur nutritionnelle des végétaux concernés, ne pas l'entamer trop tôt pour en avoir suffisamment à la mauvaise saison et notamment lors de la pousse, incertaine, de la nouvelle herbe (Lemaire, 2008; Andrieu $\&$ al., 2008). Différentes cultures de l'herbe se dégagent, comme le pointe Antoine Doré $\&$ al dans ce 
Thema à propos du Vercors : intensifier la production d'herbe - grâce à des fertilisants pour avoir du « volume » ou favoriser la qualité des fourrages. La saisonnalité structure l'inscription de l'élevage dans les territoires, selon un calendrier et des circuits précis qui alternent déploiement puis repli des bêtes dans l'espace pastoral, avec parfois stockage puis usage de fourrages (Guérin \& al., 1985; Klein \& al., 2014). Autre possibilité, le déplacement continu du troupeau selon des oscillations saisonnières qui prennent une grande diversité de formes et d'amplitude que Gérard Lenclud et François Pernet ont décrite pour l'élevage corse, en en montrant très minutieusement l'intelligence des agencements socio-écologiques (Lenclud \& Pernet, 1978). Le pâturage est bien l'expression et le produit d'un rapport professionnel à la nature. Celle-ci est l'objet autant que l'acteur d'un travail et d'une économie: les éleveurs la suivent, l'anticipent, la subissent, la transforment, la cultivent, la produisent, l'aménagent. Au-delà du profit, il ne s'agit pas seulement de sécuriser, une activité qui est de plus en plus contrainte et soumise à de forts aléas, et pour laquelle la flexibilité est une donne fondamentale (Dedieu $\&$ al., 2008). Le pâturage est aussi un partage de la nature qu'hommes et bêtes expérimentent en y cohabitant, à laquelle ils s'adaptent au fils des jours et des saisons.

De fait, «travailleurs de la nature » (Salmona, 1994), éleveurs et bergers le sont assurément. Fondée sur une étroite relation avec le vivant, animal et végétal, l'activité d'élevage fait appel à des savoirs propres. Les cultures professionnelles associées à ces élevages à l'herbe (ou à la feuille, ou au lichen...) reposent notamment sur des apprentissages complexes, longs à acquérir, transmis par les prédécesseurs, faits d'observations, de communications, d'intuitions, de sensations, d'interactions. De tels savoirs renvoient aux Traditionnal Ecological Knowledge (TEK) (Berkes, 2007), aux « savoirs naturalistes locaux « (Bérard E al., 2005), ou encore aux « savoirs paysans » (Dupré G., 1991). Ces appellations ne sont pas complètement synonymes, mais au-delà de leur sens propre et des questions que certains de leurs termes (autochtonie, traditionnel) peuvent poser, elles ont en commun de pointer d'une part, l'intelligence technique et sociale des rapports aux milieux et aux ressources naturelles, et d'autre part, leur capacité adaptative. C'est une véritable culture - au sens anthropologique du terme - des interactions hommes/ animaux/milieux naturels/ressources qui se construit, et dont des travaux très documentés à la croisée de l'éthologie, de l'écologie et de la zootechnie ont montré la complexité ainsi que la grande richesse des régimes cognitifs sur lesquels elles reposent (Meuret, 2010; Roturier \& Roué dans ce volume; Ingold 1980, Lécrivain \& al., 2013; Baumont, 2006; pour n'en citer que quelques-uns). Cette reconnaissance n'existe cependant pas partout. M. Fernanda de Torres nous le rappelle sans équivoque: en Uruguay, les ingénieurs dénient aux camperos leurs savoirs propres, les uns et les autres s'inscrivant dans des rapports au monde vivant opposés, développant des pratiques et conceptions de la prairie, antagonistes.

\section{... à l'épreuve de leurs modernisations}

L’amélioration génétique et la chimie révolutionnent la productivité fourragère, tandis que surgissent des nouveaux procédés, nouveaux équipements, nouvelles incitations et règles (Vissac, 2006). Ces évolutions peuvent se traduire tant par l'intensification fourragère jusqu'au « zéro pâturage » comme dans le Livradois-Forez, ou encore par la mobilisation d'anciennes ressources alimentaires délaissées depuis la motorisation de 
l'agriculture, et redécouvertes face aux incertitudes climatiques, aux coûts de l'aliment industriel. Des espaces hier interstitiels et intermédiaires sont ainsi sollicités à nouveau, qu'il s'agisse des fonds de vallées cévenoles, des estives ou des coteaux pyrénéens (Gibon, 2005). Ces marges fourragères ne sont pas les plus productives sur le plan agronomique, mais peuvent assumer un rôle décisif dans la complexité croissante des calendriers et des allotements. Ces lieux de soudure, lieux de bien être, lieu de diversification et de sécurisation alimentaire redessinent une géographie fourragère de plus en plus riche, précieuse aussi pour sécuriser et s'adapter, face aux changements climatiques notamment (Duru \& al., 2001; Charbonneau, 2008). Leurs pratiques ne sont donc pas immuables et ancestrales: elles demandent une actualisation, voire la redécouverte de savoirs, dont la transmission a possiblement été interrompue.

En faisant pâturer, le berger ou le « campero » exploite le milieu et dans le même temps le nettoie, l'entretient, le protège des incendies: il en prend soin. Reconnaitre la contribution de l'élevage à l'entretien des milieux, à la lutte contre les incendies et les avalanches, à la production de paysages (Ambroise \& Deffontaines, 2008), à l'entretien des milieux humides (Bouchard \& al., 2003) est un véritable enjeu. En Europe, depuis les années 1980, les politiques publiques suivent cette tendance (Alphandéry $\&$ Billaud Dir., 1996), mais ce n'est guère le cas ailleurs, au Sud où l'élevage est plutôt associé à une menace pour les écosystèmes (désertification, érosion, déforestation). Lorsqu'il n'est pas exposé à cette critique, il est en quelque sorte naturalisé et nié en tant qu'activité économique et professionnelle. Le campero « produit » autant des bovins qu'un milieu remarquable, la pampa, laquelle en retour cultive les hommes. Expert du « campo natural », faisant corps avec le milieu dans l'exercice de son métier comme dans son nom, la reconnaissance de ses savoirs se heurte aux points de vue de l'agronome. Se pose finalement la question de la légitimité des répertoires cognitifs et de la grille d'évaluation à laquelle ces derniers sont soumis (Figuié \& Hubert, 2012). Qui évalue quoi et comment? À quelle échelle? L'éleveur a ses objectifs, ses contraintes, ses exigences et ses cadres spatio-temporels. D'autres points de vue s'expriment, extérieurs aux mondes de l'élevage, qui formulent de nouvelles questions, pointent des enjeux vifs, simultanément et à d'autres échelles de temps et d'espaces.

\section{... jusqu'aux « global change » et « global cow»}

L'impératif environnemental a donné corps à un nouveau champ scientifique : les « global earth sciences ». Celles-ci s'emploient à définir et évaluer l'impact des activités humaines sur le changement global, notamment climatique, appréhendé cette fois-ci à l'échelle mondiale. D’autres échelles, d'autres outils, bien éloignés des éleveurs, sont ici en vigueur, nous plaçant dans un monde de flux chimiques et services écosystémiques planétarisés. La prairie dite "permanente », c'est-à-dire celle qui n'est pas labourée et semée régulièrement, est un objet important de ces nouvelles interrogations et espoirs (Lemaire $\mathcal{E}$ al., 2005). Mais il n'est plus seulement question de parcelles ni d'exploitations agricoles, de parcours, d'estive ou de prairies cultivées. Il n'est plus uniquement question de qualités fourragères et de bien-être animal, il ne s'agit plus de produire des fromages de qualité, de l'agneau à l'herbe ou de la laine. C'est en d'autres termes que l'élevage est considéré: on y parle de stockage de carbone, émissions de méthane et autres émanations sulfurées, voire sulfureuses. Il faut en particulier atténuer la production de gaz à effet de serre, changement climatique oblige (Lemaire \& al., 2011; Soussana \& Duru, 2007). 


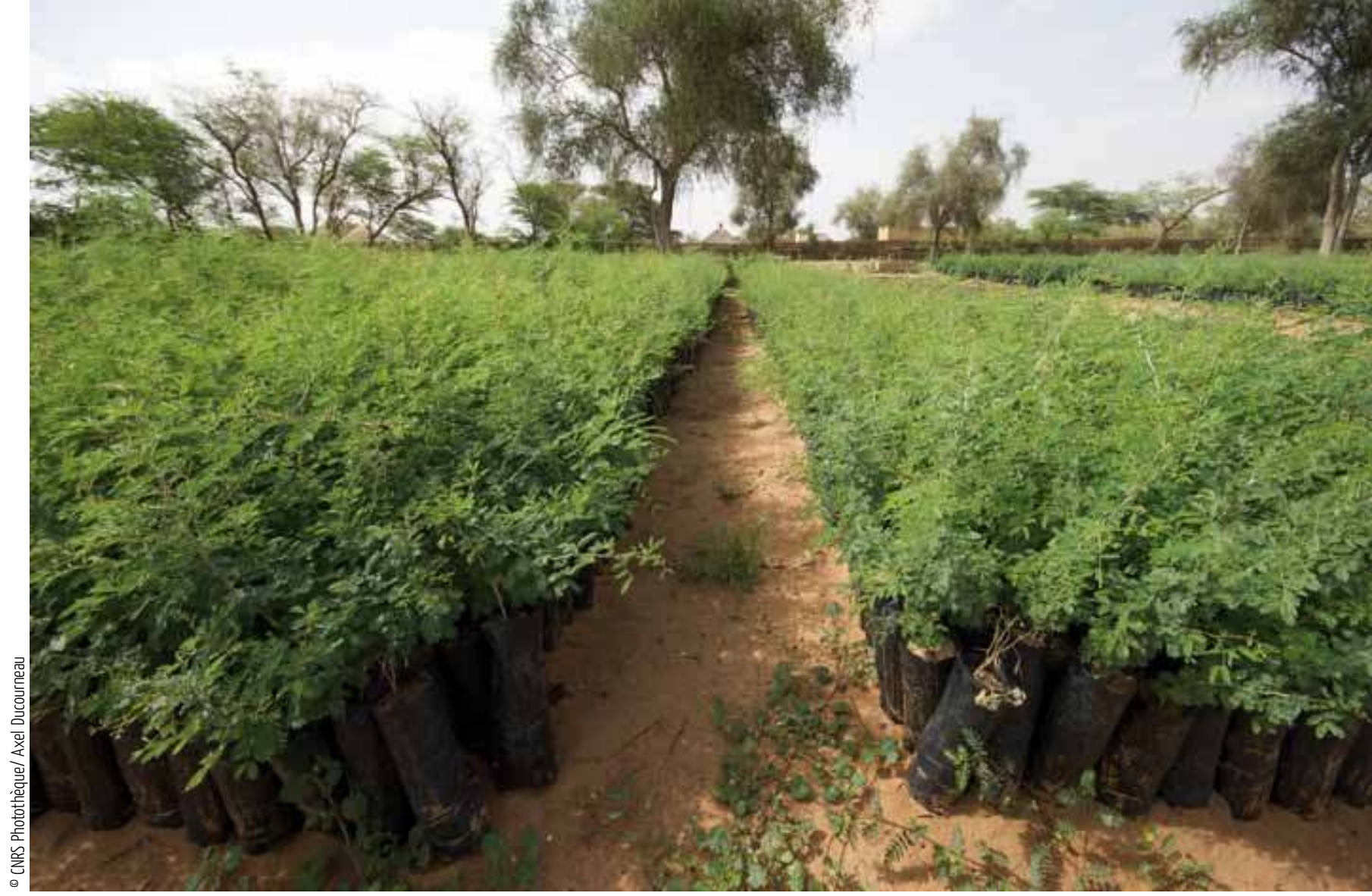

Si les éleveurs sur prairies et parcours souffrent partout du changement climatique (Allard \& Balent, 2007), les activés d'élevage de ruminants y contribuent également en émettant des Gaz à Effet de Serres (GES): du méthane par la fermentation entérique dans la panse, de l'oxyde nitreux dans leurs déjections. Ils contribuent aussi indirectement à des émissions de dioxyde de carbone lors des déforestations pour ouvrir de nouveaux pâturages. Ces éléments sont, logiquement, retenus à charge contre le secteur (Steinfeld, 2006). Le rôle des activités agricoles, et de l'élevage en particulier, se trouve de fait au cœur de ces débats sur le changement climatique. Et c'est sans doute la plus grande nouveauté relativement aux années 1960. L'élevage y est vertement critiqué; nous le disions en introduction. Mais lui et les pâturages peuvent aussi constituer une planche de salut. En effet, venant contrebalancer ce rôle négatif lié à l'émission de GES, un nouveau point de vue se fait jour, renvoyant à un champ de connaissances complexe, qui mesure la contribution du pâturage pour «stocker », « piéger », « séquestrer » du carbone dans les sols (Parson $\&$ al., 2011; Blanfort $\&$ al., 2011; Soussana $\mathcal{E}$ al., 2004; Soussana $\&$ al., 2010), ou encore à éviter des pertes par le feu accidentel (Nepstad $\&$ al., 2001). S'ouvre ainsi depuis quelques années une économie des flux d'éléments chimiques et de matières organiques entre les composants des agro-écosystèmes, à différentes échelles spatiales depuis les micro-organismes du sol jusqu'au jardin planétaire. Ainsi, de la même façon que l'élevage entretient des territoires, certains pâturages contribuent à la production de services écosystémiques (Lemaire, 2013).

La nécessité de prendre du recul et d'envisager l'élevage d'un point de vue global, l'impératif du changement climatique ainsi que la prise en compte des « fonctions prairiales » mettent en tension local et global, deux échelles et façons de penser très différentes. Elles ne doivent pas pour autant faire disparaître ni la vache au champ, ni l'éleveur au

Les pépinières d'acacias de la Grande Muraille Verte sont préparées dès le mois de mai pour que les plants soient transférables sur les parcelles dès les premières pluies. Ici, les pousses ont 3 mois. Elles seront ensuite arrosées régulièrement par les habitants, au long d'une immense bande qui s'étend de Dakar à Djibouti: l'objectif est de freiner l'avancée vers le Sud du Sahara, et de ses dunes qui étouffent progressivement les pâturages sahéliens. Un tel projet n'est pas sans faire écho à la restauration des terrains de montagne qu'a connue la France à partir du xixe siècle. 
travail, ni le territoire spécifique où ils s'inscrivent, derrière une « global cow » telle celle que J. van der Ploeg décrit à propos de l'élevage hollandais: un animal réduit au flux d'azote qu'il produit (van der Ploeg, 2008). Défini à l'échelle nationale, le taux de production d'azote est devenu un indicateur de développement durable qui permet d'évaluer les élevages par la seule comptabilité de leurs bêtes - et le sociologue de préciser qu'un seul outil est alors requis: l'hélicoptère. On ne peut que regretter avec lui l'étroitesse du point de vue sur le développement durable comme sur l'élevage, qui est ici exprimé. La vache y est-elle encore seulement un animal? Pour autant qu'il soit affecté et gouverné par des changements globaux auxquels il contribue également fortement, l'élevage herbager nous rappelle qu'il demeure encore bien un art de localités - pour reprendre, en la mettant au pluriel, la célèbre expression du père de l'agronomie moderne Olivier de Serres ([1600] 2001) à propos de l'agriculture. Nature financiarisée et cotée en bourse, biobanque, marché du carbone... Dans ce « biopoly » planétaire, les éleveurs et leurs pâturages ne sont-ils pas à la fois les premiers concernés et les moins associés aux débats dans lesquels se joue portant aussi leur avenir? D'autres formes d'évaluations s'imposent, d'autres critères et indicateurs apparaissent, qui sans doute éloignent les éleveurs un peu plus du développement durable. Entre tertiarisation de l'élevage, financiarisation de la nature, verdissement de l'économie et mondialisation des marchés, l'emboîtement des échelles spatiales apparaît plus que jamais à la fois nécessaire mais difficile à négocier pour que l'élevage ne soit pas vidé de son sens, et quittés par ses hommes, pour qu'il continue à faire société et à habiter les territoires.

\section{Le pâturage et les dynamiques d'usages des sols}

L'élevage au pâturage offre une image de permanence et stabilité trompeuse : il doit faire face à des changements d'occupation des sols cultivés et de dynamiques des espaces naturels pâturés qui sont parfois sujets à des changements importants et brusques. L'équilibre apparent ne va donc pas de soi. On peut avancer deux raisons. D'un côté, l'élevage n'est souvent pas la seule forme d'occupation du sol: ceci rend l'accès aux ressources naturelles souvent instable, remis en cause, et parfois objet d'une véritable lutte pour la place dans le territoire entre différents groupes socio-professionnels (Lussault, 2009). Les dynamiques d'occupation du sol pointent l'importance particulière de la forêt dans la redéfinition de la place et des formes de l'élevage dans les territoires. De l'autre, l'élevage à l'herbe dépend largement des conditions naturelles, elles-mêmes sujettes à de fortes perturbations.

\section{Élevages et forêts: deux mondes en dispute...}

Lorsqu'on parle de pâturage, l'arbre n'est jamais bien loin, parfois ressource alimentaire pour les troupeaux, sinon obstacle forestier à l'expansion des prairies, ou concurrent pour l'accès à la lumière, l'eau ou aux nutriments du sol (Balent $\&$ Deconchat, 2008). Cette relation ambivalente transparaît nettement dans les différentes contributions du Thema. Ce constat ravive l'intérêt d'une analyse des liens entre ces deux formes d'occupation 
du sol, lesquels n'ont cessé de structurer les histoires agraires du monde, illustrés ici de l'Équateur (Amazonie) aux latitudes extrêmes de Laponie et Patagonie. Forêts et pâturages constituent deux mondes distincts juridiquement, socialement, économiquement, politiquement. Ils ont chacun leurs acteurs, leurs institutions, leurs outils, leurs règles, leurs savoirs, leurs rationalités. Leurs rapports au temps aussi sont contrastés: le temps long prédomine pour la forêt, tandis que le temps rond, cyclique et saisonnier, gouverne les pâturages. C'est donc surtout dans la dimension spatiale que ces deux mondes se rencontrent, créant rapports de force, tensions, exclusions ou complémentarités si structurants des territoires d'élevage, et déterminants pour leur devenir. Les relations entre pâturages et forêts sont aussi anciennes que complexes. Anciennes d'abord, car l'histoire agraire est celle d'un défrichement. Beaucoup des actuelles zones de pâturages ont été gagnées sur des forêts ou des peuplements denses de ligneux arbustifs. Et inversement, comme le rappelait Pierre Deffontaines, « toute décrue humaine » entraîne un « recru forestier » (Deffontaines, 1933 : 31). Complexe, comme le montre ce Thema, à travers de multiples exemples de substitutions forêts - pâturages (et réciproquement), de dynamiques liées à des flux et reflux des prairies et donc des hommes, sur les espaces concernés... Dans le Livradois-Forez, la forêt dit le retrait de l'homme, encercle les prés et émiette le paysage agraire. En Uruguay, ce n'est pas tant l'homme qui déserte que l'industrie papetière qui convoite les pâturas de l'élevage pour y planter ses eucalyptus, dans un processus dit de reforestación. L’Amazonie est l'exemple le plus médiatisé et démesuré de la mise en pâturage des forêts primaires (Poccard-Chapuis, 2004 ; Tavarella, 2011). Depuis la fin du XIXe siècle, dans les Alpes françaises, non sans débats, y compris au sein même de l'institution forestière (Ponchelet, 1995), les forestiers ont âprement protégé les forêts contre la dent des troupeaux: il s'agissait de « restaurer la montagne », d'éviter ruissellement et érosion en laissant s'installer les forêts tout en favorisant l'engazonnement à travers un programme national très ambitieux appelé la Restauration des Terrains de Montagne (RTM). La forêt s'est alors développée sur des espaces de culture et de parcours de moins en moins pâturés, où la pression humaine s'était affaiblie, entraînant d'autres risques, d'incendie notamment dans la partie méridionale du massif. Curieux renversement de l'histoire, les politiques publiques soutiennent maintenant le pâturage qui permet dans ces territoires de montagne, de lutter contre la «fermeture des milieux ». Ces situations illustrent la transition forestière décrite par Mahler (1992), et montrent à quel point cette théorie géographique qui décrit une alternance « déforestation - reforestation » en fonction du niveau de développement des pays, repose aussi sur les expansions et rétractions des pâturages.

\section{... Mais potentiellement complémentaires}

En 1953, Jean Giono met en scène Elzéard Bouffier, un berger qui, au sortir de la première guerre mondiale, dans les Alpes, « plantait des arbres » chaque jour dans sa solitude silencieuse, semant ainsi les promesses d'une harmonie sociale et écologique que l'union à venir de l'arbre et du mouton incarne (Giono, [1953] 2014). Faut-il voir dans ce texte un manifeste sylvo-pastoral? La complexité des liens entre forêts et pâturages ne se résume pas à ces seuls rapports de concurrence, d'alternance, de substitutions dans l'espace et le temps. Arbres et fourrages sont en effet parfois pensés de concert. Ainsi, au Maroc ou en Laponie, les pratiques d'alimentation des animaux reposent « traditionnellement » 
les modalités du partage des ressources et des espaces; ils font controverse sur la place de chaque groupe social et son usage des ressources sur lesquelles on reviendra dans le dernier temps de cet article. La question de l'accès aux ressources et donc celle du droit de pacage, comme l'a pointé Olivier Barrière, apparaît fondamentale car elle rend possible - ou impossible - ces éventuelles complémentarités, elle les encourage ou les contrarie. Celles-ci se doublent d'une tension tout à fait fondamentale, entre intérêt privé et intérêt collectif, conduisant les éleveurs Samis à se tourner vers la justice, comme le signalent Samuel Roturier et Marie Roué.

Une question se pose alors, de taille: comment coordonner les groupes sociaux autour d'un usage commun de ressources que chacun voudrait s'approprier prioritairement? Les exemples ne manquent pas dans ce Thema, nous conduisant au Maroc, au Brésil, en Cévennes... De façon générique, la problématique foncière sous-jacente s'exporte sur le plan juridique, où la complexité du droit du pâturage fait l'objet de controverses, mais aussi propose des solutions comme le code pastoral malien, établissant des règles pour les pistes à bétail en réponse aux conflits qui opposent éleveurs et pasteurs. En outre, les sociétés dans leur ensemble formulent de nouvelles exigences, par exemple au Brésil vis-à-vis des fronts pionniers amazoniens, ou encore pour l'entretien des paysages ruraux français. Des éleveurs innovants peuvent trouver dans ces recompositions de nouveaux espaces, opportunités, ressources économiques et territoriales. Dans les Cévennes, les vallées abandonnées, aux prés envahis de ligneux, offrirent aux nouveaux ruraux sortis des villes, une opportunité unique d'accéder à la terre et d'y reconstruire une offre fourragère, qu'ils parviendront à valoriser dans l'AOC fromagère du Pélardon (Napoléone E al., dans ce Thema).

Partout, l'inscription spatiale de l'élevage reste plus complexe que pour les autres usages du sol, de par la mobilité inhérente à cette activité (Lasseur \& al., 2010). Elle suppose des proximités de parcelles ou pâtures, des réseaux de transport, qui sont autant de motifs à conflits, à difficultés. Criante en Afrique subsaharienne où elle peut rendre vulnérable des populations et territoires entiers (Blanc-Pamard \& Boutrais, 2006), cette problématique s'exprime aussi en Auvergne. Au cœur de la plus grande prairie de France, des éleveurs choisissent l'élevage hors-sol pour éviter (et s'éviter) à leurs vaches laitières les déplacements biquotidiens d'un pré à l'autre (Boisdon $\&$ al., dans ce Thema).

\section{... et le labourage en arbitre}

Nous avons insisté sur les relations entre élevage et forêts en montrant comment elles sont particulièrement structurantes mais les dynamiques d'occupation des sols ne jouent pas entre ces deux seuls secteurs. Au sein même du monde agricole, des formes s'opposent et se disputent également le territoire. Il existe par exemple d'autres façons d'exercer l'élevage associées à une alimentation issue de cultures intensives, ailleurs ou ici, nécessitant d'importants intrants chimiques. C'est ainsi que la pampa argentine ou la savane brésilienne sont dévorées par la monoculture du soja, la plupart du temps transgénique, nourriture « moderne » d'un bétail rapidement élevé à l'autre bout du monde, possiblement confiné en bâtiment, qui le mange sous forme de tourteaux. Monoculture préoccupante en termes écologiques, mené au pas des marchés internationaux, qui vient concurrencer l'élevage dans ses formes les plus herbagères et ses territoires les plus fragiles ou singuliers comme l'est le biome "pampa ». Il en est de 
même en Europe, avec le retournement des prairies permanentes, où on a considéré qu'il était économiquement préférable de remplir les auges des vaches laitières d'ensilage de maïs plutôt que de les nourrir à l'herbe. Enfin, il n'est pas inutile de rappeler, comme le pointe Claudine Friedberg, que « même le paisible mouton, symbole d'une agriculture en accord avec la nature » (Friedberg, $2000: 111$ ), dément parfois sa réputation et rappelle que sa dent peut être dure. Car l'espèce est une chose, le système d'élevage et les formes qu'il prend en sont une autre, tout aussi déterminantes. Le ranching, dans certaines de ses formes, est particulièrement visé. Basé sur l'importation de races et de technologies étrangères, connecté avec les marchés internationaux, le ranching est un véritable outil de conquête socio-économique des territoires dont les effets dévastateurs sur l'écosystème sont immenses. La Patagonie argentine offre un bon exemple, où pâturage rime avec surpâturage. Comme le rappellent Fernando Coronato et Jean-François Tourrand, l'élevage ovin pour la laine commença par chasser les premiers occupants indigènes; il s'est ensuite massivement déployé, dans une dynamique d'accaparement foncier, sans autre souci de la ressource que celui d'en tirer le meilleur profit immédiat, conduisant à l'exploitation minière du sol et de la végétation, dégradant d'immenses territoires dans un processus proche de la désertification. Autre exemple de lutte pour la place: l'histoire agraire de la Nouvelle-Zélande est bien celle d'une lutte et d'une occupation pastorale aux dépens de la population maorie et de l'agriculture (Wood \& al., 2008). À une tout autre échelle, William Blanc montre dans le Curiosa, en quoi la circulation répétée des troupeaux transhumants est une forme d'occupation du territoire provençal, alliant savoir et pouvoir, dont le vaste réseau de « drailles » témoigne aujourd'hui.

On le voit, les récents changements globaux affectent fortement la dynamique spatiale des pâturages. Dans la sphère économique, les filières se réorganisent spatialement en fonction de la logistique globalisée et des avantages compétitifs propres à chaque territoire. Ce faisant, elles modifient la géographie des productions, notamment la géographie de l'élevage (Delgado \& al., 1999; Steinfeld \& al., 2010), générant spécialisations ou déprises, concentrations (clusters) et ceintures (Poccard-Chapuis $\&$ al., 2011). Fortement dépendant de coûts spatialisés (coût de la terre, coût du transport, accès à l'énergie électrique et à la chaîne du froid), l'élevage ne peut que réorganiser ses pâturages en conséquence. Comment résister à de tels drivers (Gerber $\&$ al., 2010) ? Comment prendre le temps du pâturage quand ce sont les marchés internationaux approvisionnés en viande générique qui donnent le tempo? Un bœuf élevé sur la pampa est abattu vers trois ans. La complémentation alimentaire à base de soja permet de gagner un an, et de s'affranchir de la saisonnalité fourragère (Vissac, 2006). Au cœur des enjeux de l'élevage, il y a bien là des questions de temps à accorder, de rythmes à tenir ou à accélérer.

Enfin, il est un dernier point qu'il nous faut évoquer, ne serait-ce que brièvement. $\mathrm{Au}$-delà de la mondialisation économique, la sphère environnementale intervient aussi fortement dans cette réorganisation. Le potentiel fourrager des pâturages est modifié par le changement climatique et entraîne de nouvelles localisations, parfois à l'échelle des biomes comme en Afrique où le Sahara étend son emprise vers le Sud et y repousse l'élevage sahélien, parfois avec des sécheresses qui rendent l'activité plus ou moins risquée, affectant directement son développement. Les pâturages sont aussi ravagés par la multiplication des incendies, en particulier sous climats tropicaux et méditerranéens. Par ailleurs, les réglementations prévues pour faire face à ces dérèglements globaux valorisent une nouvelle fonction fondamentale de l'élevage, la capacité à atténuer les émissions de gaz à effet de serre. Les politiques de protection de la biodiversité ne sont pas en reste, 
privilégiant soit l'établissement de réserves foncières face à l'expansion agricole (land sparing), ou encore des mosaïques d'aires agricoles et aires protégées (land sharing) (Torquebiau, 2012). Face à tous ces moteurs de localisations, délocalisations, concurrences et synergies, la localisation des pâturages est aujourd'hui bien éloignée du modèle classique «Ager - Saltuts - Sylva » théorisé par les Romains, ou même de celui de l'économiste Von Thünen (1851) où pâturage rime avec éloignement, utilisation extensive, voire réserves foncières, de terres arables. Le poids des distances et localisations n'est plus le même, tant sont fréquents, parfois brutaux et complexes, les facteurs de changements d'usages des sols. Dans ce cadre global d'incertitudes mondialisées et de désordres localisés (et réciproquement), le territoire apparaît comme l'entité où s'organisent les nécessaires adaptations, équilibres et régulations, où s'élaborent parfois de possibles résolutions ou apaisement face aux tensions.

\section{Une fabrique sociale des territoires}

En posant « la tragédie des communs » et promouvant comme seule issue, la privatisation des parcours pour favoriser leur bonne gestion, Hardin pointe, par défaut, la nécessité d'élargir le cadre d'analyse de ces activités. Il s'agit de traiter de leur inscription dans un espace d'interactions sociales pourvoyeuses de solution pour sa gestion durable. Ces dimensions ont depuis largement été développées et théorisées en particulier de manière très emblématique par les travaux d'Elinor Ostrom (2012). L'inscription du pâturage dans les territoires se réaffirme ainsi comme un objet d'étude porté par la définition des enjeux futurs pour l'élevage (Herrero, 2013). Le pâturage apparaît alors comme une composante particulière de l'intégration agriculture - territoire qu'on peut analyser selon trois dimensions : 1) les conditions, essentiellement juridiques, d'accès aux ressources et l'incidence sur les conditions de subsistance des familles rurales en particulier les plus pauvres; 2) les modalités de définition, en particulier en regard des caractéristiques des produits de l'élevage de ce qui dans un territoire fait ressource pour cet élevage; 3) les convergences/divergences entre les formes que revêt l'activité d'élevage et des attentes qui lui sont adressées par la communauté locale ou une société plus englobante.

\section{Les régulations de l'accès aux espaces pâturés}

Les obstacles à la circulation du bétail et des hommes mettent en échec les modes d'élevage à l'herbe. En effet, le pâturage est un mode d'exploitation des milieux qui, comme le relève Olivier Barrière, ne présuppose pas de la maîtrise totale du sol (contrairement à la culture). Le pâturage s'accommode dans une certaine mesure, de relatives formes de précarités foncières. Cette précarité apparaît à Fernandez-Gimenez (2002) inhérente à la nature même de l'activité. L'auteure pointe ainsi la difficulté de définir les limites spatiales et sociales des ressources et des groupes sociaux car la flexibilité, tant sociale que spatiale, est une composante intrinsèque et essentielle du système pastoral (Dedieu $\varepsilon$ al., 2008). Le bon équilibre entre sécurité et maintien de la flexibilité constitue de fait un défi important à l'adresse des politiques de régulations foncières pour qu'elles 
permettent de conforter réellement cette activité. Il s'agit ainsi de reconnaître que certains espaces sont des composantes indispensables aux systèmes pastoraux tout en acceptant que le rapport de la société locale au droit foncier y soit défini et reconnu de manière relativement souple et évolutive.

De ce fait, les dispositifs d'action publique régulant l'accès foncier s'avèrent d'autant plus nécessaires au maintien du pâturage que le contexte actuel est caractérisé par une concurrence pour l'usage des terres, accrue par le développement de cultures dédiée à la production énergétique. Un mouvement d'« accaparement des terres » s'est développé au cours de la dernière décennie particulièrement en Afrique et en Asie du Sud Est. Celui-ci vise souvent des espaces qualifiés par les instances nationales des pays concernés, de « sous utilisés » ou « abandonnés » (Borras \& Franco, 2012). Les espaces pastoraux dont on vient de rappeler les spécificités foncières, y figurent en bonne place. Ce changement d'affectation prive alors des populations rurales d'espaces indispensables à leur subsistance. C'est donc l'usage pastoral selon des règles reconnues au sein de la société locale qui est remis en cause. Or, l'intégration de populations rurales socialement et économiquement vulnérables repose sur ces mêmes zones de pâturage. Dans de tels contextes, maintenir l'activité de pâturage est aussi une question de lutte contre la pauvreté en milieu rural particulièrement dans les pays du Sud (Ancey \& al., Dir. 2013, Duteurtre $\&$ Faye, Dir. 2009). De fait, les politiques publiques - comme l'ont été les politiques de développement appelant à la sédentarisation - limitant les conditions

En Afrique, le gardiennage est une tâche souvent confiée aux enfants et

aux femmes.

Au Lesotho, jeune berger surveilleant un troupeau collectif de village.

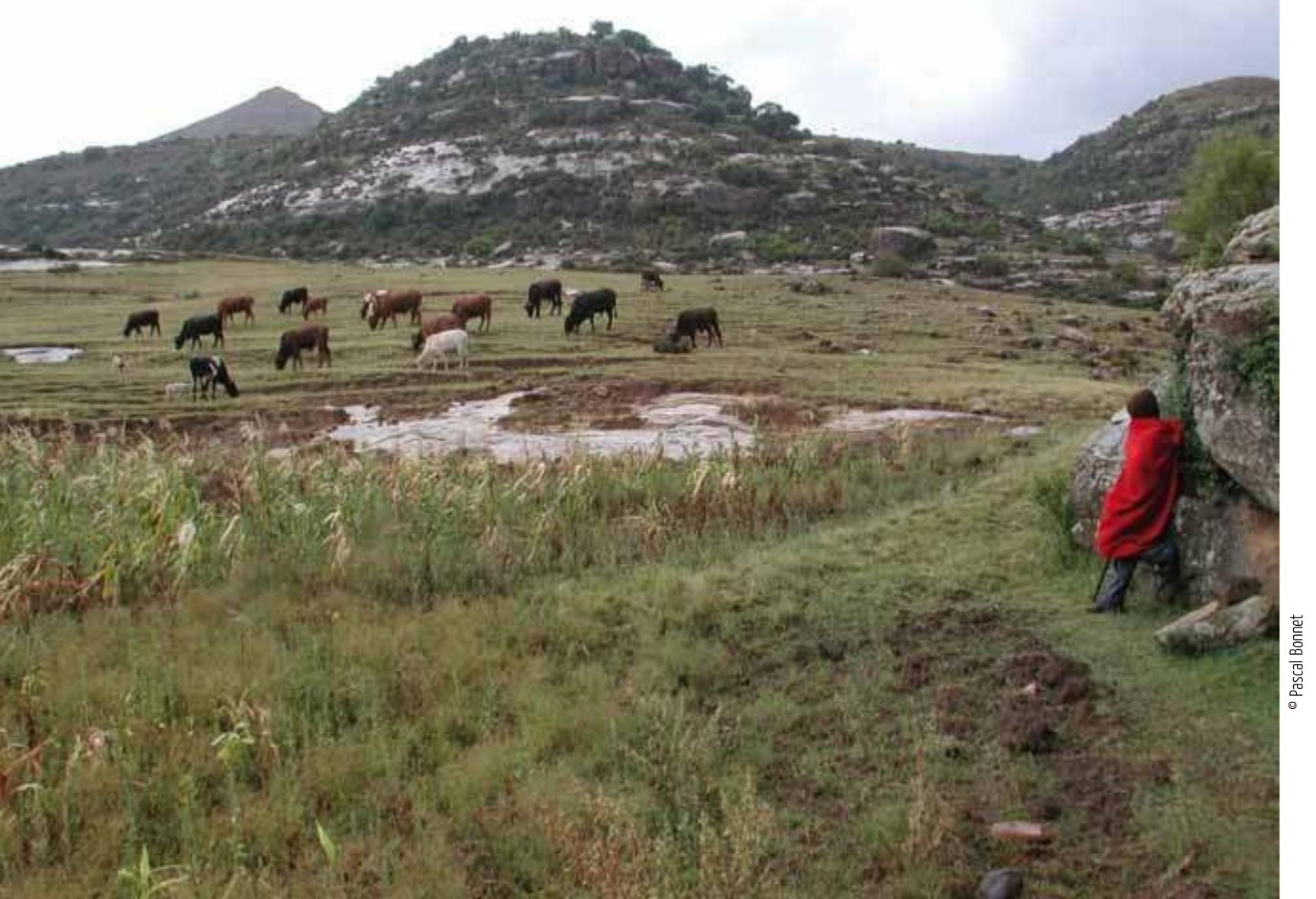


de circulation des hommes et des bêtes sont souvent synonymes de paupérisation et de vulnérabilités sociale et économique, comme cela a été observé notamment en Iran (Digard \& Pâpoli-Yazdi, 2008). De même, de nombreux dispositifs de protection des écosystèmes, en restreignant les conditions d'accès aux espaces, souvent à l'adresse de pasteurs, pointent la délicate articulation entre les enjeux de préservation environnementale, l'accès foncier et la vulnérabilité sociale, trois dimensions dont l'articulation relève d'une très grande complexité (Sternberg \& Chatty Dir. 2013; Parayil \& Tong 1998; Hoelle 2015). En Mongolie, la décollectivisation des terres liée à la chute du communisme s'est accompagnée d'une redistribution (inégale) des terres et des moyens de production. La " privatisation » de l'utilisation des parcours et des campements ${ }^{2}$ utilisés lors des déplacements saisonniers, conjuguée à l'accroissement des cheptels, ont occasionné surpâturage, désorganisation et inégalités sociales - que le régime antérieur n'était toutefois pas à même d'empêcher (Devienne, 2013). Ailleurs, nombreux sont les exemples soulignant la possibilité de combiner un accès collectif à des ressources non privatisées avec la préservation des écosystèmes et le développement des activités humaines. Ainsi, loin d'être associés à une tragédie fatale, les formes collectives de gestion de pâturages, également collectifs, peuvent aussi être une garantie contre le surpâturage et la dégradation des ressources fourragères (Mac Bea, 1990; Moritz $\&$ al., 2003). Ils ont aussi un avenir (Harvey, 2011) mais celui-ci ne peut-être que le produit d'une construction collective produite aussi à l'échelle des territoires et impliquant la pluralité des acteurs et des groupes sociaux dont la vie y est liée.

\section{Ressources territoriales et pâturage}

La construction de ce qui fait ressource constitue un puissant levier d'intégration des acteurs de l'élevage à l'échelle d'un territoire. Martine Napoléone \& al., montrent en quoi la réponse se trouve parfois au cœur de discussions collectives visant d'une part, à définir dans les collectifs professionnels les pratiques et modalités de mobilisation des ressources s'insérant dans un système de normes locales élaborées entre pairs (Darré, 1999), et d'autre part à produire un argumentaire au sein de la filière d'élaboration des produits de l'élevage portant sur les spécificités de ces produits. Ainsi, dans le Vercors et les Cévennes, le clivage entre les « laitiers » et les « fermiers » renvoie à deux façons de pratiquer l'élevage, de se définir et de se démarquer, voire s'opposer au sein d'un monde professionnel, par et dans l'usage de la ressource fourragère. Maintenir une relation entre les spécificités d'un espace, les ressources qu'il pourrait recéler et les caractéristiques des produits alimentaires issus de l'agriculture, est une voie largement explorée comme vecteur de développement local, en particulier à travers la promotion de systèmes agricoles localisés (Muchnik \& de Sainte Marie, Dir. 2010). Concernant les produits de l'élevage, l'origine de l'alimentation des animaux et le recours au pâturage figurent en bonne place dans l'affirmation de ces spécificités. Ils constituent par ailleurs un puissant vecteur d'activation de ressources territoriales (Roux $\mathcal{E}$ al., 2006) importantes pour l'identité et le développement de certains territoires. Pour leur part, Nicolas Lacombe et François Casabianca insistent dans ce Thema sur les conflits d'usage et de légitimité liés à l'exploitation de certaines ressources pastorales convoitées par d'autres groupes sociaux, dans le cadre de démarches de qualité qui deviennent « concurrentes». 
La typicité de certains produits de l'élevage tient notamment à la richesse et la composition floristique des fourrages qui font des espaces concernés un élément clé de différenciation sociale, culturelle et économique. D’une part, cette différenciation permet de mobiliser le marché pour tenir face à la concurrence de produits animaux issus de formes d'élevage plus industrialisées ou de la compétition pour l'usage des terres (Sylvander $E$ al., 2008). D'autre part, elle contribue à la reconnaissance culturelle de produits dits « de terroir » par laquelle s'exprime, s'objective et se protège un lien au lieu et à l'histoire (Faure, 1999; Bérard \& Marchenay, 1995). De fait, les coordinations dans l'espace et dans le temps de ces ressources hétérogènes naturelles, culturelles, cognitives, techniques par laquelle un groupe se distingue, voire prend sa revanche, économiquement mais aussi socialement et culturellement, relèvent de dynamiques patrimoniales par lesquelles, de difficile à marginal, le territoire s'en trouve requalifié; il devient singulier et porteur d'une certaine richesse (Dupré, 2000). Ce travail de caractérisation de la typicité s'opère selon des cadres juridiques et scientifiques bien éprouvés, en Europe notamment. Dans les pays du Sud, ces derniers sont peut-être moins présents, mais ils n'en offrent pas moins de possibles dynamiques permettant d'articuler valorisation, reconnaissance et protection des produits et des territoires, dont Claire Cerdan et Delphine Vitrolles offrent un exemple à propos de l'élevage sur pampa en Argentine (Cerdan \& Vitrolles, 2008). Ces entreprises ne reposent pas seulement sur des savoirs d'éleveurs: elles mobilisent aussi largement des savoirs de biologistes permettant de mettre en évidence la façon dont la qualité des prairies (notamment leur composition et diversité floristiques) détermine en partie la qualité des produits, laitiers ou carnés (Coulon, 2008). Cela nous situe encore à une autre échelle du vivant: bactériologique, chimique. Les populations animales ont été relativement peu évoquées tout au long de ce Thema. Il est bien évident que la gestion du capital génétique et des aptitudes animales sont une dimension décisive dans la pratique du pâturage et dans la différenciation des produits (Lauvie \& al., 2011, Béranger \& al., 2008).

Mise en œuvre sur de vastes espaces - qui pour être non nécessairement privatisés n'en sont pas moins appropriés - support de multi-usages et fortement structurante des paysages, l'activité de pâturage est porteuse d'interactions sociales larges. Les connexions aux attentes d'autres acteurs et d'articulations à ces attentes s'expriment au-delà de la communauté professionnelle. Elles font sortir le pâturage des exploitations d'élevage et de son monde professionnel. Il produit des biens collectifs, appropriés et valorisés à une échelle large, en termes d'image ou de tourisme. Il se rend visible et se partage, non plus seulement dans le conflit, dimension sur laquelle nous avons beaucoup insistée tant elle est fondamentale dans les processus d'entre-définition des groupes sociaux. Le pâturage, et avec lui ses produits, génère, en Uruguay comme au Maroc ou dans le Vercors, une sociabilité élargie, institutionnalisée et souvent festive. La dimension festive est marquée et repose sur une « consommation » conjointe des produits et des territoires, faisant de la fête un outil de reconnaissance et d'affirmation « pour les autres » d'une particularité locale (Dupré, 2005). À ce niveau englobant, se gouvernent les intérêts et initiatives des territoires, qui voient se retrouver d'autres groupes sociaux, y compris dans de débats vifs, comme on l'a vu. À une vision d'une communauté rurale définissant en autonomie ce qui constitue ses ressources et les modalités de son exploitation, se substitue une vision impliquant un ensemble large d'acteurs dont des « tiers » distants qui s'impliquent fortement dans la définition des normes et conditions d'usage souhaitables. Les sociétés dans leur ensemble reformulent des exigences, expriment de nouvelles attentes, par 
exemple au Brésil vis-à-vis des fronts pionniers amazoniens, ou encore pour l'entretien des paysages ruraux français. La construction sociale des territoires par le pâturage prend des formes bien différentes. Entre la Patagonie, où l'interaction frise le collapsus de ce pays du mouton après épuisement de la ressource fourragère, au tourisme du Vercors qui s'approprie des pratiques pastorales pour les ériger en fête estivale, l'Amazonie parie sur l'économie verte pour dynamiser ses bassins pastoraux et propose en ce sens des dispositifs de certification territoriale. Les interactions les plus abouties valident des formes de certification de produits alimentaires (indications géographiques), de systèmes techniques (bonnes pratiques environnementales, etc.) associées à des arènes élargies, apportant une certaine forme de reconnaissance, de stabilité technique et juridique, et accompagnant l'émergence d'alternatives aux lois d'un marché mondialisé. Mais tous les pays ne partagent pas la même expérience de ces dispositifs; plus récente au Sud en ce qui concerne les produits animaux. En revanche partout, elles sont coûteuses en temps. Or, le temps, ou plus exactement ses rythmes, constituent bien un des éléments clés de ces diverses dynamiques. En effet, nous l'avons rappelé, certains territoires sont marqués par l'instabilité et de rapides changements et réorientations économiques ou politiques. Ainsi, le libéralisme économique est souvent à la source d'une dégradation aussi rapide qu'irréversible de l'équilibre entre activités d'élevage et renouvellement des ressources naturelles en particulier. Ceci accompagnant une dérégulation des systèmes de production fatale à la gestion des milieux pastoraux comme à la structure sociale de l'élevage.

\section{La politisation territoriale de l'élevage}

L'émergence des territoires comme espaces de décisions et de gestion peut permettre de mieux valoriser les nouveaux intérêts locaux construits autour du pâturage. L'activité d'élevage peut y puiser la reconnaissance de ses multiples fonctions, en même temps que le territoire y trouver une nouvelle ressource. C'est à cette échelle que peut s'opérer la reformulation - ou la traduction - d'enjeux globaux portés souvent en des termes qui échappent complètement aux éleveurs. La légitimation de l'activité des pasteurs, activité par définition très exposée, s'en trouve ainsi confrontée à des points de vue diversifiés. Le territoire peut offrir la scène locale où s'effectue un travail d'argumentation et de justification des pratiques d'élevage dont on a dit qu'elles étaient susceptibles d'être remises en question et en cause à d'autres échelles d'analyse. Les pasteurs se trouvent ainsi d'autant plus vulnérables que l'échelle d'analyse est globale, générant des rapports de force inégaux incriminant des processus exprimés à des échelles globales face à des connaissances d'éleveurs par définition fortement situées, renforçant ainsi les dissymétries au détriment des pasteurs. Pensons par exemple, pour reprendre l'exemple de la

« global cow » décrit précédemment, aux émissions de gaz à effet de serre comptabilisés à l'échelle planétaire. Quelle argumentation pourrait fournir un pasteur sur la qualité de sa production si celle-ci s'évalue en grammes de carbone émis par kilo de produit, faisant fi de ses autres caractéristiques? Quels arguments peut-il développer sur des relations élevage/environnement si celles-ci se mesurent en regard d'une liste d'organismes vivants classés à l'échelle d'un pays ou d'un continent? L'échelle territoriale permet de nuancer et de reformuler ces liens en en déplaçant le cadre d'analyse.

Un ensemble de dispositifs liés à des politiques publiques encadre et oriente l'activité, contribuant à la redéfinir. Le pâturage est souvent cantonné aux « territoires difficiles » 
ou aux marges des territoires centraux - le Thema le souligne fortement, soit actuellement (Patagonie, Amazonie, Laponie), soit au cours de leur histoire agraire (les alpages français, complètement marginalisés jusqu'aux années soixante-dix, en sont une bonne illustration). Pourtant, dans ces mêmes zones, l'émergence des territoires comme espaces de décision et de gestion a redonné un intérêt aux pâturages. Sous le coup de politiques publiques spécifiques, le pâturage puise une reconnaissance de ses multiples fonctions, en même temps que le territoire trouve une nouvelle ressource économique assurant son peuplement et son développement contrastant avec la situation marginale qu'il a pu connaître par ailleurs. C'est ce principe qui rend l'Amazonie attractive aux yeux des éleveurs du Centre Sud brésilien. À l'échelle des grands massifs montagneux européens, c'est cette volonté de préservation « d'espaces en herbe » (Dobremez \& al., 2014) qui a motivé le dispositif de soutien à l'élevage: « prime à la vache tondeuse » dans ses premières déclinaisons nationales puis « Indemnité Compensatrice de Handicap Naturel » dans sa version européenne récente (Catan, 2014). Tout un pan de l'élevage européen est ainsi soutenu pour continuer, au-delà de ses fonctions de productions de biens alimentaires, à fournir de ce qu'on appelle maintenant couramment des « services » environnementaux largement évoqués dans le premier temps de cet article. Le maintien de la biodiversité, la structuration des paysages, l'ouverture des milieux, la réduction des risques d'incendies, la régulation hydrique, renvoient vers un nouveau champ de recherches et de légitimation réapproprié localement, éventuellement à la base d'un véritable contrat entre éleveurs et collectivités locales. Il s'agit de contribuer à l'action publique en proposant des évaluations des activités et de leurs transformations. Le défi consiste à renforcer la cohérence de ces diverses incitations, injonctions aux différentes échelles ou elles opèrent. Mais aussi d'anticiper en maintenant les propriétés d'adaptabilité aux incertitudes actuelles et futures, locales et globales.

\section{Conduite des bêtes, ressources naturelles et gouvernement des hommes}

Si l'on considère l'histoire longue de l'élevage et de sa modernisation, force est de constater que la capacité des hommes à exploiter la nature a longtemps servi de critère distinctif pour établir le degré d'évolution (entendre de modernité, c'est-à-dire de capacité à s'inscrire dans une économie de marché) d'une société. Dans une telle optique, le pâturage est demeuré fortement associé à une économie de cueillette, implicitement relégué à ce qu'on ne considérait que comme un « simple prélèvement » de ressources naturelles spontanément renouvelables, et critiquée pour cette raison: pas de travail de l'homme, pas de maîtrise sur la nature, pas de recherche de productivité possible, et de profit et donc pas d'insertion dans une économie marchande (sans parler de mondialisation). Dans les pays développés, la modernisation de l'élevage s'est accompagnée de la définition et de la légitimation d'un système fourrager caractérisé par la culture intensive de l'herbe, sa récolte et son stockage, constituant la « nécessaire révolution fourragère » dont Pierre Chazal et l'économiste agricole René Dumont ont été les promoteurs (Chazal $\&$ Dumont, 1954). Dans cette logique d'optimisation, l'affaire est entendue: le pâturage 


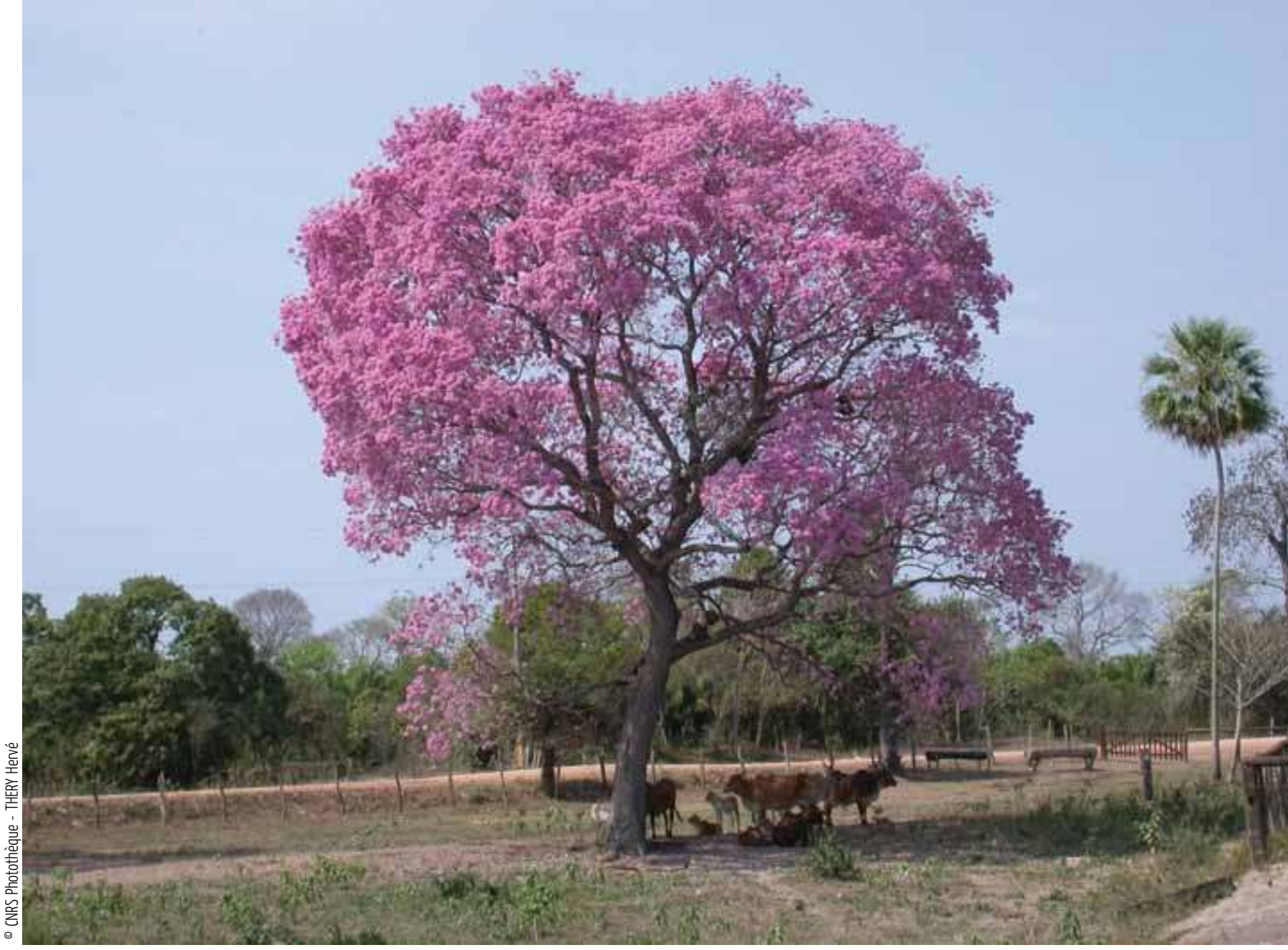

n'est un recours, une ressource, qu'en situation de mécanisation trop difficile, d'espaces et de formes d'agriculture marginalisées. Il est réduit à « une surface souvent ridiculement petite par rapport aux besoins du troupeau, surface qui manifestement joue le rôle d'un alibi symbolique (« c'est pour les promener ») beaucoup plus que celui d'une ressource alimentaire significative » (Landais 1996 : 64). Il faut attendre les grandes crises énergétiques (chocs pétroliers), l'augmentation des coûts de mécanisation et des engrais pour que le pâturage regagne quelques soutiens et quelque intérêt et ne soit plus considéré comme un pis-aller.

L'agriculture industrielle a porté des « fruits amers », nombreux, qui ont empoisonné le monde, et les agriculteurs les premiers (Robin, 2014). Les conséquences d'un élevage industrialisé, déterritorialisé, jusqu'à faire de l'herbe une exception alimentaire pour les animaux, se sont traduites en autant de crises: crises sanitaires, crises écologiques, crise de confiance chez le consommateur, crises économiques pour les éleveurs. La crise de la vache folle, nous le rappelions en introduction de ce Thema, a été sans doute l'élément décisif d'une prise de conscience de ces excès, de ces dangers, de ces impasses liées à l'industrialisation de l'élevage. Telle aura été « la leçon de sagesse » que les vaches folles nous ont adressée à cette occasion (Lévi-Strauss, 1996). Et voilà qu'on reparle du pâturage, pas uniquement comme une solution de repli face à ces crises agglomérées, mais comme une forme d'avenir pour l'élevage, qui permette de répondre aux enjeux, aux attentes et aux défis auxquels sont respectivement et ensemble confrontés les éleveurs à l'herbe, les territoires difficiles, les milieux vulnérables et la société. Car tout l'enjeu du

\section{Repos des bêtes}

L'arbre est un élément naturel important pour l'élevage. Outre un appoint de fourrage, son ombre est précieuse pour le troupeau et les éleveurs. Au bord de la route qui traverse le Pantanal, quelques bêtes d'un troupeau en transit se reposent sous un ipê rose. Près de Poconé, Mato Grosso, Brésil. Etude des impacts environnementaux de l'occupation du Pantanal. 
développement durable non seulement des activés agricoles mais aussi des territoires est bien non seulement de ne pas dissocier ces perspectives mais de les faire tenir ensemble sans établir de hiérarchie entre elles, en les considérant toutes trois justiciables d'un même intérêt, d'une même nécessité. Et la particularité du pâturage est d'être, au-delà d'une technique d'alimentation animale, une pratique où nombre de tensions se rejoignent en clé de voûte, où s'articulent échelles, de temps et d'espaces, jeux d'acteurs dont on a aperçu ici la complexité, lieux où culture et nature ne font plus qu'un.

Avec le pâturage, l'élevage ne se referme pas sur lui-même: il se pense à plusieurs, il s'ouvre sur d'autres mondes sociaux, politiques, juridiques, scientifiques, susceptibles de contribuer en retour à la reconnaissance sociale et économique des territoires d'élevage. En effet, si l'élevage vient au territoire par le pâturage, le pâturage est une façon pour les éleveurs d'y faire société - y compris dans des dynamiques de conflits. Il n'est pas qu'une histoire d'éleveurs ou d'élevage. Le mouton, la vache, le renne, la chèvre sont autant d'éléments d'une grammaire paysagère et culturelle, qui suscitent souvent, au-delà d'un corps de métier, un attachement fort et durable, un lien et un lieu de reconnaissance pour une société à la fois locale et élargie. Ils peuplent les territoires et derrière eux, et avec eux, se trouvent des hommes au travail dont Pierre Lieutaghi, dans la riche préface qu'il consacre au Théatre d'agriculture et ménage des champs, fait à juste titre remarquer qu'ils tendent à ne plus y être visibles, à s'y faire de plus en plus rares (Lieutaghi, 2001), à ne plus, finalement, habiter les territoires.

L'élevage herbager apparaît sous certaines conditions, possiblement porteur d'une nouvelle modernité qui viendrait prendre le contre-pied de la révolution verte et du productivisme mondialisé que cette dernière a généré. Une telle perspective questionne de façon centrale la capacité de l'élevage et de sa composante pastorale à contribuer à la maîtrise, sur des pas de temps long, de la coévolution des milieux et des activités humaines qui s'y inscrivent. Les enjeux ne sont pas seulement économiques, ou plutôt, ils ne peuvent être qu'indissociablement sociaux et écologiques. Car le pâturage, on l'a montré, est autant une ressource culturelle, agronomique et sociale qu'alimentaire: milieux, techniques et sociétés étant dans un même continuum constituant la trame de ce « tissu sans couture » (Hughes, 1983) par lequel un fait technique ne peut être que dans le même temps social (Simondon, 2012; Latour \& Lemonnier, 1994; Akrich, 1994); et politique. L'inclusion sociale d'éleveurs autrement marginalisés ou renvoyés à d'autres activités et d'autres lieux, la contribution de l'élevage herbager à la fabrique et au développement de territoires habités, la production d'une diversité de produits alimentaires de qualité, qui garantissent le principe même du vivant : -sa reproductibilité-, sont bel et bien des enjeux majeurs. L'enjeu d'un véritable développement durable réside dans la possibilité d'articuler ces nombreux domaines autour d'approches coordonnées (Lucas, 2012 : 36) dont la difficulté tient sans doute à la pluralité des échelles, et des rythmes impliqués. Ceux du vivant d'abord: de la vie microbienne au cycle du carbone, en passant par la vie d'une vache au pré ou les dynamiques de végétation, le temps ne s'organise pas de la même manière. Ceux des politiques publiques, du contrat quinquennal, dont la reconduction est incertaine, à la nécessité d'accompagner les transformations profondes des systèmes de production agricole à une échelle pluri décennale en acceptant l'incertitude des perspectives (Hubert, 2002). Ceux de l'élevage et des éleveurs bien entendu: des tâches effectuées quotidiennement aux objectifs annuels, en passant par l'inscription dans le temps générationnel qui suppose - à tout le moins questionne - la transmission de l'exploitation agricole. À chacune de ces échelles, un monde social se déploie avec ses 
experts, ses savoirs, ses enjeux qui entrent en possible contradiction, sinon en conflits, les uns avec les autres. L'avenir des éleveurs comme celui des prairies, qui n'ont de permanentes que le nom ${ }^{3}$, dépendent aussi de dynamiques très structurantes portées par des forces, notamment économiques, sinon financières, en partie extérieures à ses mondes ou qui en occupent parfois désormais le cour. L'élevage à l'herbe nous dit à la fois la difficulté mais aussi l'intérêt, de prendre le temps du pâturage. Il supporte ces diverses tensions que l'impératif de «nourrir la planète » « sans la détruire pour pouvoir le faire encore longtemps », exacerbe encore davantage.

Encore faudrait-il que ce noble objectif ne soit pas un encouragement à un productivisme sans limite (Rosin, 2012; Tittonell, 2014) qui ne ferait qu’appauvrir et épuiser les éleveurs, leurs animaux et les milieux qu'ils peuplent et produisent ensemble. En cela, le maintien et le développement durable de l'élevage sur pâturage dans ses territoires ne peuvent aujourd'hui se penser indépendamment d'un projet de société plus global, dans lequel la question économique ravive au plus haut point la redéfinition de notre rapport aux ressources naturelles et la nécessité de les penser selon des formes garantissant l'inclusion et la sécurisation sociale des éleveurs. Il s'agit bien de travailler à un «futur commun », commun, aux hommes et à la planète, et ne fasse pas mentir le nom du rapport que nous avons évoqué en introduction, le « Rapport Brundtland ». Le combat à mener est-il « contre la vache » tant qu'on ne la laissera pas «paître en paix dans les lieux écologiquement et socialement compatibles »? (Friedberg, 2000 : 111), ou contre la toute-puissance des marchés internationaux (Trouvé, 2015)? À quelles échelles de temps, d'espaces mais aussi d'intérêts faut-il penser les choses? Comment, c'est-àdire de quelle façon, combiner la pluralité des mondes qui interviennent directement ou indirectement dans le pâturage, et que réciproquement, le pâturage nourrit et cultive en retour? Les interrogations et les difficultés sont nombreuses. Elles vont bien au-delà de la seule question du pâturage car les enjeux excèdent le seul monde de l'élevage et sonnent comme autant de défis adressés aux communautés scientifiques dont une large palette disciplinaire se trouve ici convoquée. Les paradigmes candidats à proposer des alternatives existent. " Dans le grand marché des mots pour penser le changement » (Ollivier \& Bellon, 2013: 173), l'un d'eux est aujourd'hui beaucoup utilisé : agroécologie. La notion ne renvoie toutefois pas à un seul et unique projet. Son usage recouvre en effet une diversité de « courants » et de propositions, dont certaines prennent particulièrement au sérieux la nécessité de faire tenir ensemble et sur un même plan, les exigences économiques, environnementales et sociales, appelant en conséquence à une refonte, elle aussi en profondeur, des paradigmes scientifiques (Altieri, 2002).

Ce travail a été conduit dans le cadre du projet de recherches MOUVE financé par l'Agence nationale de la recherche (projet ANR-2010-STRA-005-01). 


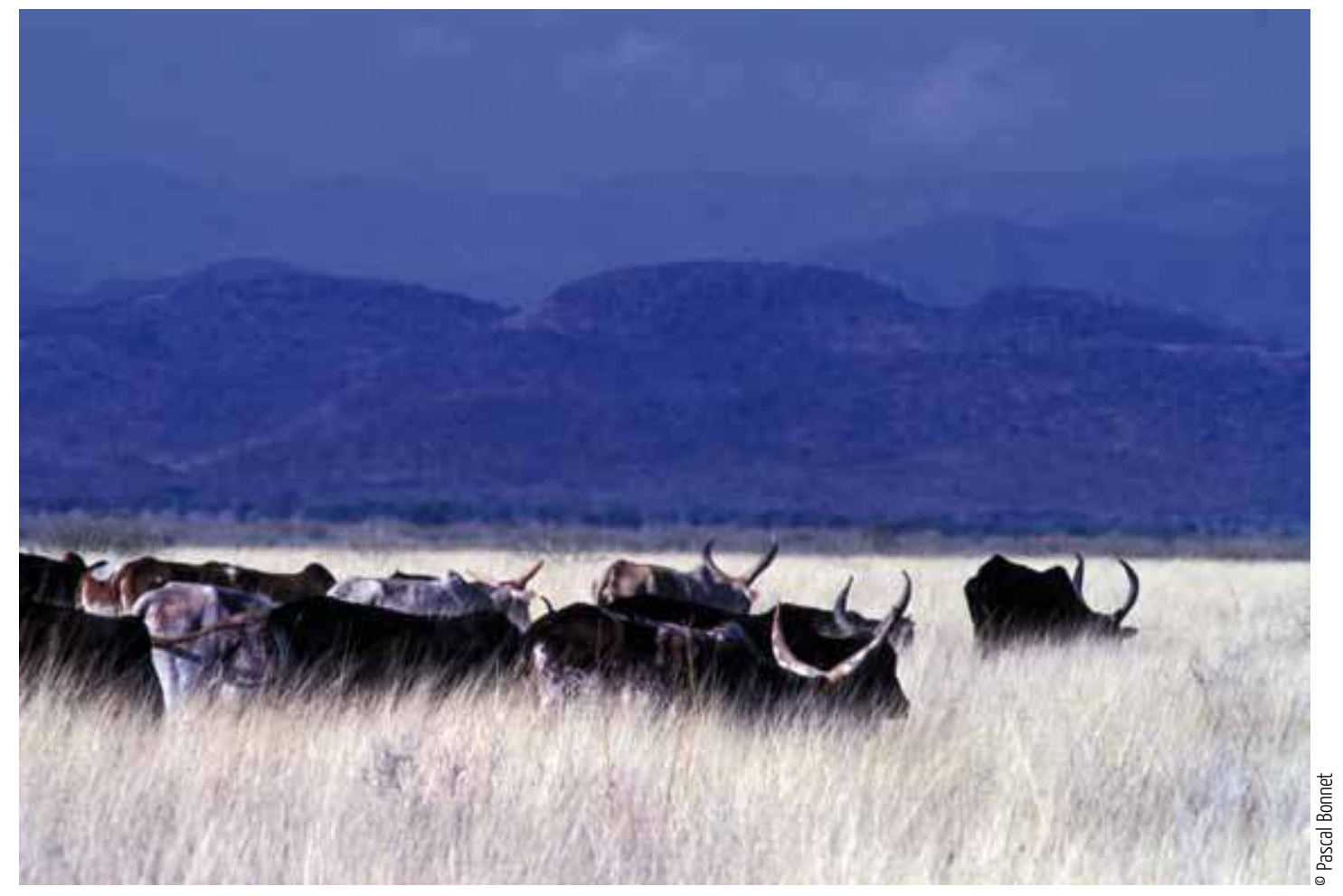

\section{Ranching}

L'activité de gardiennage de troupeau peut-être une activité socialement valorisée. En Éthiopie dans une plaine sédimentaire fertile, zone de transhummance en saison des pluies au pied de l'escarpement des hauts plateaux abyssins, la garde de ce troupeau de lignage de zébu de race afar est confiée à des hommes adultes. 


\section{POUR CITER CET ARTILLE:}

Dupré, L., Lasseur, J. \& R. Poccard-Chapuis (Dir.) 2015 Faire pâturer, faire société, durablement, In Pâturages Techniques \& Culture 63: 202-231.

\section{NOTES}

Photo d'ouverture: Moutons à Marseille. Lorsque la ville ne va pas au pâturage, c'est le mouton qui s'invite en ville. Ici à Marseille, en juillet 2013, la transhumance ovine célèbre la capitale européenne de la culture et parade sur les quais. Les éco-moutons parisiens ou les bars à moutons coréens disent la nécessité de restaurer un lien à l'animal dont les herbivores sont des emblèmes.

1. Par exemple, dans le rapport Livestock Long Shadow il est principalement question d'élevage mais pas tellement d'éleveurs: on compte 1530 occurrences pour le terme «élevage », 72 pour « éleveurs » (Steinfeld, 2006).

2. La maîtrise du bâti agricole est parfois déterminante dans l'accès aux ressources fourragères, comme le relèvent Doré \& al., dans ce Thema: dans les Pyrénées où la propriété d'une grange foraine va de pair avec l'accès aux ressources herbagères d'altitude.

3. La catégorie des «prairies permanentes»se distingue pour l'agronome des prairies qui sont régulièrement retournées pour être renouvelées ou intégrées à des successions culturales (ces dernières sont appelées «prairies artificielles » si elles sont majoritairement ensemencées en légumineuses, « prairies temporaires » si elles sont majoritairement ensemencées en graminées). La réglementation agricole française retient la durée minimale de six années pour qualifier une prairie de permanente. Les prairies naturelles qui font l'objet de peu d'intensification des pratiques agricoles, et qui sont donc reconnues pour leur intérêt environnemental (richesse floristique), sont une composante de cette catégorie des prairies permanentes. 


\section{RÉFÉRENCES}

Akrich, M. 1994 Comment sortir de la dichotomie technique/société. In Latour, B. et Lemonnier, P. De la préhistoire aux missiles balistiques: l'intelligence sociale des techniques. Paris: La Découverte: 105-131.

Alard, D. \& Balent, G. 2007 Sécheresse: quels impacts pour la biodiversité en systèmes prairiaux et pastoraux? Fourrages 190: 197-206.

Alphandéry, P. \& Billaud, J.-P. (Dir.) 1996 Cultiver la nature, Études rurales, 141-142.

Altieri, M.-A. 2002 Agroecology: the science of natural resource management for poor farmers in marginal environments, Agriculture, ecosystems \& environments 93 (1-3) : 1-24.

Ambroise, R. \& Deffontaines, J.-P. 2008 Prairies et paysages. In Béranger C., Bonnemaire J. (Dir.) Prairies, herbivores, territoires: quels enjeux? Paris: Quae Éditions: 37-69.

Ancey, V., Avelange, I. \& Dedieu, B. (Dir.) 2013 Agir en situation d'incertitude en agriculture. Regards pluridisciplinaires au Nord et au Sud. Bruxelles, Bern, Berlin, Frankfurt am Main, New York, Oxford, Wien: Peter Lang.

Andrieu, N., Coléno, F., Duru, M. 2008 L'Organisation du système fourrager source de flexibilité face aux variations climatiques. In B. Dedieu \& al. (Dir.) L'Élevage en mouvement. Flexibilité et adaptation des exploitations agricoles d'herbivores. Paris: Quae Éditions: 95-110.

Balent, G. \& Deconchat, M. 2008 Le Paysage à l'interface des activités agricoles et forestières: Une introduction, Revue Forestière Française LX (5) : 515-524.

Beaumont, I. 2006 Que fabrique le pastoralisme? des agneaux, des bergers, de la montagne. Bordeaux: Association Française de Sociologie (Colloque de l'Association Française de Sociologie).

Béranger, C., Bonnemaire, J. 2008 Prairies, herbivores, territoires: quels enjeux? Paris: Quae Éditions.

Béranger, $\mathrm{Cl}$. \& al. 2008 Conséquences zootechniques d'une orientation herbagère prédominante. In Béranger C., Bonnemaire J. (Dir.) Prairies, herbivores, territoires: quels enjeux? Paris: Quae Éditions: $117-128$

Bérard, L. \& Marchenay, Ph. 1995 Lieux, temps et preuves. La construction sociale des produits de terroir, Terrain 24: 153-164.

Bérard, L. \& al. (textes réunis par) 2005 Biodiversité et savoirs locaux naturalistes en France. Paris: coédition IDDRI, IFB, INRA, CIRAD.

Berkès, F. 2007 Understanding uncertainty and reducing vulnerability: lessons from resilience thinking, Natural hazards41: 283-295.

Blanc-Pamard, C. \& Boutrais, J. 2006 Thème et variations. Nouvelles recherches rurales au Sud. Paris : ORSTOM.

Blanfort, V. \& al. 2011 Impacts et services environnementaux de l'élevage en régions chaudes, Productions animales 24 (1): 89-112.

Borras, S.M. Jr, \& Franco, J.-C. 2012 Global land grabing and trajectories of agrarian change : a preliminary analysis, Journal of agrarian change 12 (1): 34-59.

Bouchard, V. \& al. 2003 Sheep grazing as management tool in Western European saltmarshes, CR Biologies 326, Supplement $1: 148-157$.

Brisebarre, A. M., Fabre P., Lebaudy, G. 2009 Sciences sociales, regards sur le pastoralisme contemporain en France. Lirac: La Cardère Éditeur.

Brisebarre, A.-M. 1978 Bergers des Cévennes. Histoire et ethnographie de l'élevage ovin et de la transhumance en Cévennes. Paris: Éditions Berger-Levrault.

- 2013 Chemins de transhumance. Histoires de bêtes et de bergers du voyage. Paris: Delachaux-et-Niestlé.

Caron P., Hervieu, B. \& Hubert, B. 2013 Des mondes agricoles en transformation, Natures Sciences Sociétés (21): 53-55.

Catan, A. 2014 La Préservation des prairies dans la PAC: les raisons d'une illusion, Les dossiers de l'environnement de l'INRA - Écologisation des politiques publiques et des pratiques agricoles (34): 35-50. 
Cerdan, Cl., \& Vitrolles, D. 2008 Valorisation des produits d'origine: contribution pour penser le développement durable dans la Pampa Gaúcha au Brésil, Géocarrefour 83/3: 191-200.

Charbonneau, M. 2008 De la transhumance au nomadisme: les nouveaux modes de déplacements des sociétés pastorales andines, Mappemondes 90 (2) [en ligne] http://mappemonde.mgm.fr/numl8/ articles/art08203.html. Consulté le 13 avril 2015

Chazal, P. \& Dumont, R. 1954 La Nécessaire révolution fourragère et l'expérience lyonnaise. Paris: Le journal de la France Agricole.

Coulon, J.-B. 2008 Herbe et qualité des produits animaux. In Béranger C., Bonnemaire J. (Dir.) Prairies, herbivores, territoires: quels enjeux? Paris: Quae Éditions: 49-69.

Daget, P., \& Godron, M. (Dir.) 1995 Pastoralisme: troupeaux, espaces et sociétés. HATIER, AUPELF, UREF, Universités francophones.

Darré, J.-P. 1999 La production de connaissances pour l'action. Paris: coédition MSH- INRA.

Darré, J.-P. \& al. 1994 Des raisons aux pratiques. Dialogue avec un éleveur ovin, Études rurales 131-132: 107-181.

De Serres, O. 2001 [1600] Le Théâtre de l'agriculture et mesnage des champs. Paris: Actes sud:

Dedieu, B. \& al. (Dir.) 2008 L'Élevage en mouvement. Flexibilité et adaptation des exploitations agricoles d'herbivores. Paris: Quae Éditions.

Deffontaines, P. 1933 L'Homme et la forêt. Paris: Gallimard.

Delgado, C. \& al. 1999. Livestock to 2020: the Next food revolution. IFPRI Food, Agriculture, and the Environment, Discussion Paper 28. Washington, D.C. (USA) : IFPRI.

Deverre, C., \& de Sainte Marie, C. 2008 L'Écologisation de la politique agricole européenne. Verdissement ou refondation des systèmes agro-alimentaires, Revue d'Études en Agriculture et Environnement 89: 83- 104 .

Devienne, S., 2013 Régulation de l'accès aux parcours et évolution des systèmes pastoraux en Mongolie, Études mongoles et sibériennes, centrasiatiques et tibétaines [En ligne], 43-44 | 2013, mis en ligne le 20 septembre 2013, consulté le 9 décembre 2014. URL: http://emscat.revues.org/2104.

Digard, J.-.P. \& Pâpoli-Yazdi, M.H. 2008 Le Pastoralisme mobile en Iran. Ses variantes, leurs déterminants et leurs conséquences pour le développement, Études rurales 181: 89-102.

Dobremez, L. \& al. 2014 Les Alpages sentinelles. Un dispositif original pour une nouvelle forme de gouvernance partagée face aux enjeux climatiques, Journal of alpine research 102- 2

Dupré, G. 1991 (Dir.) Introduction. In Savoirs paysans et développement. Paris: Karthala-Orstom: 17-57.

Dupré, L. 2000 Du Marron à la châtaigne d'Ardèche. La relance d’une production "traditionnelle ». Paris: CTHS.

— 2005 « Patrimonialiser: entre naturalisation et excès d'historicité » in L. Bérard \& al. (textes réunis par), Biodiversité et savoirs locaux naturalistes en France, coédité par l'IDDRI, l'IFB, l'INRA et le CIRAD: politiques publiques?

Duru, M., Hazard L., Jeangros B., \& al. 2001 Fonctionnement de la prairie pâturée: structure du couvert et biodiversité, Fourrages 2001 (166) : 165-188.

Duru, M., Papy F., Soler, L.-G. 1988 Le concept du modèle général et l'analyse du fonctionnement de l'exploitation agricole, Compte rendu de l'Académie d'agriculture de France 4 (74) : 82-93.

Duteurtre, G. \& Faye, B. (Dir.) 2009 L'Élevage, richesse des pauvres. Paris: Quae Éditions.

Even, M.-A. 2009 L'IAASTD, une expertise internationale qui marque un changement de paradigme pour l'agriculture et le développement. Paris: Ministère de l'agriculture et de la pêche (service publications et statistiques -note Prospective et évaluation, avril - n 6).

Faure, M. 1999 Un produit agricole « affiné » en objet culturel. Le fromage beaufort dans les Alpes du Nord, Terrain 33: 81-92.

Faye B. 2008 Bergers du monde. Paris: Quae Éditions.

Fernandez-Gimenez M.E. 2002 Spatial and social boundaries and the paradox of pastoral land tenure: a case study from postsocialist Mongolia, Human Ecology 30 (1) : 49-78. 
Figuié, M. \& Hubert, B. 2012 « Pour qui, pourquoi une ressource est-elle jugée dégradée? In Pâturages et agriculture familiale au Brésil, Nature Sciences Sociétés 20, 3: 297-309.

Friedberg, C., 2000 Un Combat contre la vache. In Un Terrien des îles. À propos de Jacques Barrau, Journal d'agriculture traditionnelle et de botanique appliquée 42 : 107-112.

Gerber, P. \& al. 2010. Livestock in geographical transition. In Steinfeld H., Mooney H. \& Schneider F., Neville L. Livestock in a changing landscape. Volume I: Drivers, Consequences, and Responses. Island Press; Washington DC. : 51-67.

Gibon, A. 2005 Managing grassland for production, the environment and the landscape Challenges at the farm and the landscape level, Livestock Production Science 96: 11-31.

Giono, J. 2014 [1953] L'Homme qui plantait des arbres. Paris: Poche.

Guérin, H. \& al. 1985 Les Choix alimentaires des ruminants domestiques (bovins ovins, caprins) sur les pâturages sahéliens. Leurs facteurs de variation et leurs conséquences. Damas: ACSAD et AOAD (Conférence internationale du 7-12 sept. sur les productions animales en zones arides).

Hardin, G. 1968 The Tragedy of the commons, Science 13 décembre 1968162 (3859) : 1243-1248.

Harvey, D. 2011 The Future of commons, Radical history review 109: 101-107.

Herrero, M. \& al. 2013 The Roles of livestock in developing countries, Animal 7 (1): 3 - 18.

Hoelle J. 2015 Rainforest Cowboys: the rise of ranching and cattle culture in Western Amazonia. Austin: University of Texas Press.

Hubert, B. 2002 Sustainable development; think forward and act now. Agricultures and sustainable development; the stakes of knowledge and research attitudes. INRA faced with Sustainable Development: Landmarks for the Johannesburg Conference. Paris: INRA-Éditions (les dossiers de l'environnement de l'INRA - 22).

Hughes, T. P. 1983 Networks of Power. Electrification in Western Societies, 1880-1930. Baltimore: John Hopkins University Press.

Ingold, T. 1980 Hunters, pastoralists and ranchers. Cambridge: Cambridge University Press.

Jackson, J. \& Ash, A. J. 1998 "Tree-grass relationships in open eucalypt woodlands of northeastern Australia: influence of trees on pasture productivity, forage quality and species distribution", Agroforestry systems Volume 40, Issue 2: 159-176.

Klein, H.D. \& al. 2014 Les Cultures fourragères. Collection Agricultures Tropicales en poche. Paris: Quae Éditions; CTA; Presses agronomiques de Gembloux.

Landais, E. \& Deffontaines, J.-P. 1994 L'Espace d'un berger: pratiques pastorales dans les écrins. In Blanc-Pamard, C. \& Boutrais, J. (Dir.). Dynamique des systèmes agraires: à la croisée des parcours: pasteurs, éleveurs, cultivateurs. Paris: ORSTOM: 321-335.

Landais, E. 1996 Élevage bovin et développement durable, Le courrier de l'environnement de l'INRA 29: 59- 72 .

Landais, E., Deffontaines J.-P., Benoît M. 1988 Les pratiques des agriculteurs. Point de vue sur un courant nouveau de la recherche agronomique, Études rurales 109: 125-158.

Lasseur, J. \& al. 2010 Modélisation des relations entre dynamiques des territoires et des systèmes d'élevage dans le massif du Luberon, Cahiers Agricultures 19 (2): 90-96.

Lasseur, J. 2005 Sheep farming systems and nature management of rangeland in French Mediterranean mountain areas, Livestock production science 96 (1): 87-95.

Latour, B. \& Lemonnier, P. 1994 (Dir.) De la préhistoire aux missiles balistiques. L'intelligence sociale des techniques. Paris: La Découverte.

Lauvie, A. \& al. 2011 Diversity of rare breed management program: between conservation and development, Livestock science, 140: 161-170.

Lécrivain, E. \& al. 2013 Troupeaux ovins dans des parcours embroussaillés: le comportement animal fournit de nouveaux critères pour le diagnostic pastoral, Fourrages 214: 159-168.

Lemaire, G., 2008 Sécheresse et production fourragère, Innovations Agronomiques 2, 107-123.

- 2013 Les Prairies, ressources pour l'élevage et pour l'environnement, un compromis indispensable pour l'agriculture. Paris: Académie d'Agriculture de France. 
Lemaire, G., Hodgson, J., Chabbi, A. 2011 Grassland productivity and ecosystem services. Wallingford, UK: CAB International.

Lemaire, G., Wilkins, R. \& Hogdson J. 2005 Challenges for grassland science: managing research priorities, in Agriculture, Ecosystems \& Environment 108: 99-108.

Lenclud, G. \& Pernet, F. 1978 Ressources du milieu, gestion du troupeau et évolution sociale: Le cas de la Corse, Études rurales 71/72 (Juil. Dec.): 49-87.

Lévi-Strauss, Cl., 1996 La Leçon de sagesse des vaches folles, Études rurales, 157-158: 9-14.

Lieutaghi, P. 2001 Introduction. In de De Serres O. Le théâtre de l'agriculture et mesnage des champs. Arles: Actes sud: $7-54$.

Lochet, F. 2013 « Les Pâturages de la guerre froide. Garrett Hardin et la Tragédie des communs », Revue d'Histoire Moderne et Contemporaine, 60 (1): 7-36.

Lucas, J.-M. 2012 Culture et développement durable. Il est temps d'organiser la palabre... Paris: Centre d'information et de ressources pour les musiques actuelles (Irma).

Mac Bea, J.-T. 1990 Turkana Pastoralism. A case against the tragedy of the commons, Human Ecology 18: 81-103.

Mahler, A.-S. 1992 The forest transition, Area 24 (4) : 367-379.

Maris, V. 2014 Nature à vendre. Les limites des services écosystémiques. Paris: Quae Éditions.

Mathieu, A. 2004 Conceptions des agriculteurs et modèles agronomiques. Le pâturage des vaches laitières dans le Jura, Natures Sciences Sociétés (12) 4: 387- 399.

MEA 2005 Current state and trends assessment, Washington D.C.: Island Press.

Meuret, M. (Dir.) 2010 Un Savoir-faire de berger. Paris: Quae Éditions.

Moritz, M., Sholte, P., Hamilton, I. M. \& al. 2003 Open acess, open system : pastoral managment of common - pool ressources in the Chad Basin, Human Ecology 41 ( 3) : 351-365

Muchnik, J. \& de Sainte Marie C. (Dir.) 2010 Le Temps des Syal. Vivres et territoire. Paris: Quae Éditions.

Nepstad, D. \& al. 2001 Road paving, fire regime feedbacks, and the future of Amazon forests, Forest Ecology and Management 154 (3): 395-407.

Nepstad, D. \& al. 1994 The Role of deep roots in the hydrological carbon cycles of amazonian forets and pastures, Nature 372: 666-669.

Ollivier, G. \& Bellon, S. 2013 Dynamiques des agricultures écologisées dans les communautés scientifiques internationales, Nature Sciences Sociétés 2: 166-181.

Ostrom, E. 2012 Future of the Commons: Beyond Market Failure and Government Regulations. Londres: Institue of economic affairs.

Parayil, G. \& Tong, F. 1998 Pasture-led to logging-led deforestation in the Brazilian Amazon: The dynamics of socio-environmental change, Global Environmental Change-Human and Policy Dimensions (8) 1: 63-79.

Parsons, A.-J. \& al. 2011 Primary production of grasslands, herbage accumulation and use, and impacts on climate change. In Lemaire G., Hodgson J. \& Chabbi J. (Dir.) Grassland productivity and Ecosystem Services. Wallingford UK: CAB International: 3-18.

Ploeg (van der), J. D. 2008 The New Peasantries: Struggles for Autonomy and Sustainability in an Era of Empire and Globalization. London and Sterling: Earthscan.

Poccard-Chapuis, R. 2004. Les réseaux de la conquête. Filières bovines et structuration de l'espace sur les fronts pionniers amazoniens. Université Paris X - Nanterre, France (Thèse de doctorat)

Poccard-Chapuis, R., Bonaudo, T. \& Lossouarn, J. 2011 Élevage, filières et territoires en régions chaudes, Productions animales 24 (1): 129-144.

Ponchelet. D. 1995 Le débat autour du déboisement dans le département des Basses-Alpes, France (18191849) The debate surrounding deforestation in the Basses-Alpes department, 1819-1849, Revue de géographie alpine 83 (1): 53-66.

Ravis-Giordani, G. 1983 [rééd 2001] Bergers corses. Les communautés rurales du Niolu. Ajaccio: Albiana/Parc Naturel Régional de Corse.

Robin, M.-M. 2014 Les Moissons du futur. Comment l'agroécologie peut nourrir le monde. Paris: Arte éditions 
Rosin, Ch. 2013 Food security and the justification of productivity in New-Zeeland, Journal of Rural Studies 29: $50-58$.

Roux, E., Vollet D \& Pecqueur, B. 2006 Coordination d'acteurs et valorisation des ressources territoriales. Les cas de l'Aubrac et des Baronnies, Économie Rurale 293: 20-37.

Salmona, M. 1994 Les Paysans français: Le travail, les métiers, la transmission des savoirs. Paris: L'Harmattan.

Serres (de) O. 2001 [1600] Le Théâtre de l'agriculture et mesnage des champs. Arles: Actes sud.

Simondon, G. 2012 Du Mode d'existence des objets techniques. Paris: Aubier.

Soussana, J.-F. \& al. 2004 Carbon cycling and sequestration opportunities in temperate grasslands, Soil use and Management 20: 219-230.

Soussana, J.-F. \& al. 2010 Mitigating the greenhouse gaz balance of ruminant production systems through carbon sequestration in grasslands, Animal 4 (3): 334-350.

Soussana, J.-F. \& M. Duru 2007 Grassland science in Europe facing new challenges: biodiversity and global environmental change, CAB Reviews 272: 11.

Steinfeld, H. \& al. 2006 Livestock long shadow: Environmental issues and options. Rome: FAO.

Steinfeld, H. \& al. 2010 Livestock in a changing landscape. Volume I: Drivers, Consequences, and Responses. Washington DC: Island Press.

Sylvander, B., de Fontguyon G., Sans P. 2008 Comment valoriser les qualités spécifiques des viandes bovines et ovines et des produits laitiers. In Béranger C., Bonnemaire J. (Dir.) Prairies, herbivores, territoires: quels enjeux? Paris, Quae Éditions: 91-116.

Sternberg, T. \& D. Chatty (Dir.) 2013 Modern pastoralism and conservation. Old problems, new challenges. Cambridge: The white Horse Press.

Taravella, R. 2011 Les Rouages contemporains de la déforestation en amazonie orientale. Le cas de la Terra do Meio (Parà, Brésil), IDDRI, analyses 36. [en ligne] http://www.iddri.org/Publications/Collections/ Analyses/AN_1102_deforestation\%20amazonie_taravella.pdf

Tittonell, P. 2014 Ecological intensification of agriculture - sustainable by nature, Current Opinion in Environmental Sustainability 8: 53-61.

Torquebiau, E. 2012 Introduction to the special issue: Reconciling production and conservation at the landscape scale, Journal of sustainable agriculture 36 (3): 271-274.

Trouvé, A. 2015 Les Dérives de l'agro-industrie dans: le Business est dans le pré. Paris: Fayard.

Villanueva, C. \& Ibrahim, M. 2002 Evaluación del impacto de los sistemas silvopastoriles sobre la recuperación de pasturas degradadas y su contribución en el secuestro de carbono en lecherías de altura en Costa Rica, Agroforestería en las Américas 9 (35 - 36) : 69 - 74.

Vissac, B. 2006 Les Vaches de la république. Saisons et raisons d’un chercheur citoyen. Paris: Quae Éditions.

Von Thunen, J. 1826 The Isolated State., London: Pergamon edition.

Wang, Y., J. Wang, S. Li, \& Qin, D. 2014 Vulnerability of the Tibetan Pastoral Systems to Climate and Global Change, Ecology and Society 19, $4: 8$.

Wood V., Brooking T., Perry P. 2008 Pastoralism and politics: reinterpreting contests for territory in Auckland Province, New Zealand, 1853 - 1864, Journal of Historical Geography 34 : 220-24.

\section{RÉSUMÉ}

Faire pâturer, faire société, durablement. Éleveurs et élevages, entre inscription territoriale et changements globaux Souvent tenu pour archaiqque, l'élevage au pâturage revient dans les débats et suscite un intérêt nouveau, en partie aiguisé par les crises et les impasses sanitaires, économiques et écologiques auxquelles sont confrontés les éleveurs, impasses qui sont amplifiées par la mondialisation de l'économie agricole. Revenant sur le Thema, cet article rappelle, entre Nord et Sud, la complexité et la diversité des modalités techniques du pâturage et de ses enjeux sociaux, environnementaux et économiques. Le pâturage engage l'éleveur dans un travail avec la nature qui repose sur l'organisation très contrainte d'un rapport au temps et aux ressources du territoire. Il nourrit de véritables cultures 
professionnelles, pastorales ou agro-pastorales, entièrement tournées vers le vivant. Celui-ci impose son rythme et ses incertitudes, offre ses bienfaits tandis que, entre économie et écologie, d'autres rythmes gouvernent les activités agricoles. La place des pâtures indique celle de l'élevage, et par conséquent celle des éleveurs. Elle se joue dans des dynamiques complexes d'occupation du sol, dans lesquelles la forêt, alliée ou concurrente, intervient fortement. Dans ces dynamiques, fromages, laine, agneaux ne sont pas les seuls fruits du pâturage; s'y invitent des territoires, des paysages, des sociétés locales en prise avec des tensions largement mondialisées. Contribuant à la fabrique sociale des territoires, le pâturage est politisé. Les enjeux et les critiques dont il est, ou a pu être, parfois l'objet montrent en effet qu'il excède la seule question agricole ou l'échelle locale: il fait société. Le maintien et le développement durable de l'élevage dans ses territoires, souvent fragiles, ne peuvent aujourd'hui se penser indépendamment d'un projet de société plus global, dans lequel la question économique ravive au plus haut point la nécessité de redéfinir notre rapport aux ressources naturelles, selon des formes garantissant préservation environnementale et sécurisation des éleveurs, entre ancrage local et changements globaux.

\section{ABSTRACT}

Grazing, building society... sustainably. Farmers and farming, between territorial identity and global changes Often considered as archaic, grass farming is once more a subject of debate and has spurred renewed interest, in part brought about by the health, economic and ecological crises and deadlocks faced by livestock farmers, deadlocks that are exacerbated by the globalization of the farm economy. Back to the theme of this special issue, this article provides a worldwide overview of the complexity and the diversity of the technical aspects of grazing and its social, environmental and economic stakes. In the case of grazing, the livestock farmer undertakes a commitment with nature that is based on a very tight organization in terms of time and territorial resources. Grazing sustains genuine professional, pastoral and agro-pastoral cultures, entirely geared to living organisms. It imposes its rhythm and its uncertainties as well as offering its benefits, whereas between economy and ecology, other rhythms govern agricultural activities. The place of pastureland is indicative of that of livestock farming and, consequently, of that of livestock farmers. It plays a role in the complex dynamics of land use in which the forest, ally or competitor, deeply intervenes. Cheese, wool and lambs are not the only fruits of grazing within the framework of these dynamics. Regions, landscapes and local societies in tune with widely globalized tensions also have their place. As a contributor to the social fabric of a region, grazing is politicized. The challenges and the criticisms of which it is or could have been the object reveal that it goes beyond the mere issue of farming or the local scale: it makes culture. The sustainable development and maintenance of livestock farming - often fragile - can only be considered today within a more global social context in which economic issues sharply accentuate the necessity to redefine our relationship to natural resources so as to guarantee the preservation of the environment and the security of livestock farmers, between territorial identity and global changes.

\section{MOTS-CLÉS}

politiques publiques, usage du sol, multi-échelles, éleveurs, agroécosystèmes, pratiques, culture technique, interfaces nature-société, territoires

\section{KEYWORDS}

public policy, land use, multiscale, livestock farmer, agroecosystem, practices, technical culture, nature-society interface 